\title{
A THREE-DIMENSIONAL SYMMETRY RESULT FOR A PHASE TRANSITION EQUATION IN THE GENUINELY NONLOCAL REGIME
}

\author{
SERENA DIPIERRO, ALBERTO FARINA, AND ENRICO VALDINOCI
}

AbStract. We consider bounded solutions of the nonlocal Allen-Cahn equation

$$
(-\Delta)^{s} u=u-u^{3} \quad \text { in } \mathbb{R}^{3},
$$

under the monotonicity condition $\partial_{x_{3}} u>0$ and in the genuinely nonlocal regime in which $s \in\left(0, \frac{1}{2}\right)$.

Under the limit assumptions

$$
\lim _{x_{n} \rightarrow-\infty} u\left(x^{\prime}, x_{n}\right)=-1 \quad \text { and } \quad \lim _{x_{n} \rightarrow+\infty} u\left(x^{\prime}, x_{n}\right)=1,
$$

it has been recently shown in DSV16 that $u$ is necessarily 1D, i.e. it depends only on one Euclidean variable.

The goal of this paper is to obtain a similar result without assuming such limit conditions.

This type of results can be seen as nonlocal counterparts of the celebrated conjecture formulated by Ennio De Giorgi in DG79.

\section{INTRODUCTION}

Goal of this paper is to provide a one-dimensional symmetry result for a phase transition equation in a genuinely nonlocal regime in three spatial dimensions. That is, we consider a fractional AllenCahn equation of the type

$$
(-\Delta)^{s} u=u-u^{3}
$$

in $\mathbb{R}^{3}$, with $s \in\left(0, \frac{1}{2}\right)$, and, under boundedness and monotonicity assumptions, we prove that $u$ depends only on one variable, up to a rotation.

In this setting, as customary, for $s \in(0,1)$, we consider the fractional Laplace operator defined by

$$
(-\Delta)^{s} u(x):=c_{n, s} \int_{\mathbb{R}^{n}} \frac{2 u(x)-u(x+y)-u(x-y)}{|y|^{n+2 s}} d y,
$$

with

$$
c_{n, s}:=\frac{2^{2 s-1} s \Gamma\left(\frac{n}{2}+s\right)}{\pi^{\frac{n}{2}} \Gamma(1-s)},
$$

being $\Gamma$ the Euler's Gamma Function.

Moreover, we say that $u$ is $1 \mathrm{D}$ if there exist $u_{o}: \mathbb{R} \rightarrow \mathbb{R}$ and $\omega_{o} \in S^{n-1}$ such that $u(x)=u_{o}\left(\omega_{o} \cdot x\right)$ for any $x \in \mathbb{R}^{n}$. Then, our main result in this paper is the following:

Theorem 1.1. Let $n \leqslant 3, s \in\left(0, \frac{1}{2}\right)$ and $u \in C^{2}\left(\mathbb{R}^{n},[-1,1]\right)$ be a solution of $(-\Delta)^{s} u=u-u^{3}$ in $\mathbb{R}^{n}$, with $\partial_{x_{n}} u>0$ in $\mathbb{R}^{n}$. Then, $u$ is $1 D$.

Recently, a result similar to that in Theorem 1.1 has been established in Theorem 1.4 of [DSV16], under the additional assumption that

$$
\lim _{x_{n} \rightarrow-\infty} u\left(x^{\prime}, x_{n}\right)=-1 \quad \text { and } \quad \lim _{x_{n} \rightarrow+\infty} u\left(x^{\prime}, x_{n}\right)=1 .
$$

Therefore, Theorem 1.1 here is the extension of Theorem 1.4 of [DSV16] in which it is not necessary to assume the limit condition (1.3). 
We recall that equation (1.1) represents a phase transition subject to long-range interactions, see e.g. Chapter 5 in [BV16] for a detailed description of the model. In particular, the states $u=-1$ and $u=1$ would correspond to the "pure phases" and equation (1.1) models the coexistence between intermediate phases and studies the separation between them.

At a large scale, the separation between phases is governed by the minimization of a limit interface, which can be either of local or nonlocal type, in dependence of the fractional parameter $s \in(0,1)$, with a precise bifurcation occurring at the threshold $s=\frac{1}{2}$. More precisely, as proved in [SV12, SV14], if $u$ is a local energy minimizer for equation (1.1) and $u_{\varepsilon}(x):=u(x / \varepsilon)$, as $\varepsilon \searrow 0$ we have that $u_{\varepsilon}$ approaches a "pure phase" step function with values in $\{-1,1\}$. That is, we can write, up to subsequences,

$$
\lim _{\varepsilon \searrow 0} u_{\varepsilon}=\chi_{E}-\chi_{\mathbb{R}^{n} \backslash E}
$$

and the set $E$ possesses a minimal interface criterion, depending on $s$. More precisely, in the "weakly nonlocal regime" in which $s \in\left[\frac{1}{2}, 1\right)$, the set $E$ turns out to be a local minimizer for the classical perimeter functional: in this sense, on a large scale, the weakly nonlocal regime is indistinguishable with respect to the classical case and, in spite of the fractional nature of equation (1.1), its limit interface behaves in a local fashion when $s \in\left[\frac{1}{2}, 1\right)$.

Conversely, in the "genuinely nonlocal regime" in which $s \in\left(0, \frac{1}{2}\right)$, the set $E$ turns out to be a local minimizer for the nonlocal perimeter functional which was introduced in [CRS10]. That is, the interface of long-range phase transitions when $s \in\left(0, \frac{1}{2}\right)$ preserves its nonlocal features at any arbitrarily large scale, and, as a matter of fact, the scaling properties of the associated energy functional preserve this nonlocal character as well. Needless to say, the persistence of the nonlocal properties at any scale and the somehow unpleasant scaling of the associated energies provide a number of difficulties in the analysis of long-range phase coexistence models.

In particular, symmetry properties of the solutions of equation (1.1) have been intensively studied, also in view of a celebrated conjecture by E. De Giorgi in the classical case, see [DG79]. This classical conjecture asks whether or not bounded and monotone solutions of phase transitions equations are necessarily 1D. In the fractional framework, a positive answer to this problem was known in dimension 2 (see CSM05] for the case $s=\frac{1}{2}$ and [SV09, CS15, SV13] for the full range $\left.s \in(0,1)\right)$. Also, in dimension 3, a positive answer was known only in the weakly nonlocal regimes $s=\frac{1}{2}$ and $s \in\left(\frac{1}{2}, 1\right)$, see [CC10, CC14]. See also [Sav16] for a very recent contribution about symmetry results for equation (1.1) in the weakly nonlocal regime - as a matter of fact, the lack of "good energy estimates" prevented the extension of the techniques of these articles to the strongly nonlocal regime $s \in\left(0, \frac{1}{2}\right)$. In this sense, our Theorem 1.1 aims at overcoming these difficulties, by relying on the very recent paper [DSV16], which has now taken into account the weakly nonlocal regime for equation (1.1).

After this work was completed we have also received a preliminary version of the article [CCS17, in which symmetry results for fractional Allen-Cahn equations will be obtained also in the setting of stable solutions.

The rest of the paper is organized as follows. In Section 2, we recall the notion of local minimizers and we introduce an equivalent minimization problem in an extended space: this part is rather technical, but absolutely non-standard, since the lack of decay of our solution and the strongly nonlocal condition $s \in\left(0, \frac{1}{2}\right)$ make the energy diverge, hence the standard extension methods are not available in our case and we will need to introduce a suitable energy renormalization procedure.

In Section 3, we relate stable and minimal solutions in the one-dimensional case, by relying also on some layer solution theory of [CS14.

In Section 4 we consider the profiles of the solution at infinity and we establish their minimality and symmetry properties. 
In Section 5, we discuss the minimization properties under perturbation which do not overcome the limit profiles, and in Section [6 we recover the minimality of a solution from that of its limit profiles. The proof of Theorem 1.1, is contained in Section 7.

For completeness, in Section 8 we also provide a variant of Theorem 1.1 that gives minimality and symmetry results under the assumption that the limit profiles are two-dimensional.

It is worth to point out that the setting in Sections 2 6 is very general and it applies to all the fractional powers $s \in(0,1)$, hence it can be seen as a useful tool to deal with a class of problems also in extended spaces, so to recover minimal properties of the solution from some knowledge of the limit profiles.

\section{LOCAL MiNimizers IN $\mathbb{R}^{n}$ AND EXTENDED LOCAL MINIMIZERS IN $\mathbb{R}_{+}^{n+1}$}

Equation (1.1) lies in the class of semilinear fractional equations of the type

$$
(-\Delta)^{s} u=f(u)
$$

From now on, we will denote by $f$ a bistable nonlinearity, namely, we assume that $f(-1)=f(1)=0$, and there exist $\kappa>0$ and $c_{\kappa}>0$ such that $f^{\prime}(t)<-c_{\kappa}$ for any $t \in[-1,-1+\kappa] \cup[1-\kappa, 1]$. We also assume that

$$
\int_{0}^{1} f(\sigma) d \sigma>0>\int_{-1}^{0} f(\sigma) d \sigma
$$

To ensure that the solution is sufficiently regular in our computations, we assume that

$$
f \in C_{\text {loc }}^{1, \alpha}(\mathbb{R})
$$

with $\alpha \in(0,1)$ and $\alpha>1-2 s$. We remark that, in view of (2.3) here and Lemma 4.4 in [CS14, bounded solutions of (2.1) are automatically in $C^{2}\left(\mathbb{R}^{n}\right)$, with bounded second derivatives.

The prototype for such bistable nonlinearity is, of course, the case in which $f(t)=t-t^{3}$. Also, to describe the energy framework of nonlocal phase transitions, given $s \in(0,1), v: \mathbb{R}^{n} \rightarrow \mathbb{R}$ and $\omega \subset \mathbb{R}^{n}$, we consider the functional

$$
\mathcal{F}_{\omega}(v):=\frac{c_{n, s}}{2} \iint_{Q_{\omega}} \frac{|v(x)-v(y)|^{2}}{|x-y|^{n+2 s}} d x d y+\int_{\omega} F(v(x)) d x,
$$

where

$$
Q_{\omega}:=(\omega \times \omega) \cup\left(\omega \times \omega^{c}\right) \cup\left(\omega^{c} \times \omega\right)
$$

and

$$
F(t):=-\int_{-1}^{t} f(\tau) d \tau
$$

Definition 2.1. We say that $u$ is a local minimizer if, for any $R>0$ and any $\varphi \in C_{0}^{\infty}\left(B_{R}\right)$, it holds that $\mathcal{F}_{B_{R}}(u) \leqslant \mathcal{F}_{B_{R}}(u+\varphi)$.

Now we describe an extended problem and relate its local minimization to the one in Definition 2.1 (see [CS07]). For this, we set $a:=1-2 s \in(-1,1)$ and $\mathbb{R}_{+}^{n+1}:=\mathbb{R}^{n} \times(0,+\infty)$. Then, given any $V: \mathbb{R}_{+}^{n+1} \rightarrow \mathbb{R}$ and $\Omega \subset \mathbb{R}^{n+1}$, we define

$$
\mathcal{E}_{\Omega}(V):=\frac{\tilde{c}_{n, s}}{2} \int_{\Omega^{+}} z^{a}|\nabla V(x, z)|^{2} d x d z+\int_{\Omega_{0}} F(V(x, 0)) d x,
$$

where $\Omega^{+}:=\Omega \cap \mathbb{R}_{+}^{n+1}$ and $\Omega_{0}:=\Omega \cap\{z=0\}$. Here, we used the notation $(x, z) \in \mathbb{R}^{n} \times(0,+\infty)$ to denote the variables of $\mathbb{R}_{+}^{n+1}$ and $\tilde{c}_{n, s}>0$ is a normalization constant. Given $R>0$, we also denote

$$
\mathcal{B}_{R}:=B_{R} \times(-R, R)=\left\{(x, z) \in \mathbb{R}^{n} \times \mathbb{R} \text { s.t. } x \in B_{R} \text { and }|z|<R\right\}
$$

and $\quad \mathcal{B}_{R}^{+}:=\mathcal{B}_{R} \cap\{z>0\}=B_{R} \times(0, R)=\left\{(x, z) \in \mathbb{R}^{n} \times \mathbb{R}\right.$ s.t. $x \in B_{R}$ and $\left.z \in(0, R)\right\}$. 
In this setting, we have the following notation:

Definition 2.2. We say that $U$ is an extended local minimizer if, for any $R>0$ and any $\Phi \in$ $C_{0}^{\infty}\left(\mathcal{B}_{R}\right)$, it holds that $\mathcal{E}_{\mathcal{B}_{R}}(U) \leqslant \mathcal{E}_{\mathcal{B}_{R}}(U+\Phi)$.

The reader can compare Definitions 2.1 and 2.2. Also, given $v \in L^{\infty}\left(\mathbb{R}^{n}\right)$, we consider the $a$ harmonic extension of $v$ to $\mathbb{R}_{+}^{n+1}$ as the function $E_{v}: \mathbb{R}_{+}^{n+1} \rightarrow \mathbb{R}$ obtained by convolution with the Poisson kernel of order $s$. More explicitly, we set

$$
P(x, z):=\bar{c}_{n, s} \frac{z^{2 s}}{\left(|x|^{2}+z^{2}\right)^{\frac{n+2 s}{2}}} .
$$

In this framework, $\bar{c}_{n, s}$ is a positive normalization constant such that

$$
\int_{\mathbb{R}^{n}} P(x, z) d x=1
$$

see e.g. [Buc16]. Then we set

$$
E_{v}(x, z):=\int_{\mathbb{R}^{n}} P(x-y, z) v(y) d y=\int_{\mathbb{R}^{n}} P(y, z) v(x-y) d y .
$$

We remark that when $v \in C_{0}^{\infty}\left(\mathbb{R}^{n}\right)$, the function $E_{v}$ can also be obtained by minimization of the associated Dirichlet energy, namely

$$
\inf _{\substack{V \in C_{0}^{\infty}\left(\mathbb{R}^{n+1}\right) \\ V(x, 0)=v(x)}} \int_{\mathbb{R}_{+}^{n+1}} z^{a}|\nabla V(x, z)|^{2} d x d z=\int_{\mathbb{R}_{+}^{n+1}} z^{a}\left|\nabla E_{v}(x, z)\right|^{2} d x d z
$$

see Lemma 4.3.3 in [BV16]. Nevertheless, we want to consider here the more general framework in which $v$ is bounded, but not necessarily decaying at infinity, and this will produce a number of difficulties, also due to the lack of "good" functional settings.

We also remark that the setting in (2.5) and the normalization constant $\tilde{c}_{n, s}$ are compatible with the choice of the constant in (1.2), since, for any $v \in C_{0}^{\infty}\left(\mathbb{R}^{n}\right)$,

$$
\frac{c_{n, s}}{2} \iint_{\mathbb{R}^{2 n}} \frac{|v(x)-v(y)|^{2}}{|x-y|^{n+2 s}} d x d y=\frac{\tilde{c}_{n, s}}{2} \int_{\mathbb{R}_{+}^{n+1}} z^{a}\left|\nabla E_{v}(x, z)\right|^{2} d x d z,
$$

see e.g. formula (4.3.15) in [BV16].

Since these normalization constants will not play any role in the following computations, with a slight abuse of notation, for the sake of simplicity, we just omit them in the sequel.

In our setting, for functions $v$ with no decay at infinity, formula (2.6) does not make sense, since both the terms could diverge. Nevertheless, we will be able to overcome this difficulty by an energy renormalization procedure, based on the formal substraction of the infinite energy. The rigorous details of this procedure are discussed in the following 1 result:

\footnotetext{
${ }^{1}$ We observe that Proposition 2.3 here is also related to the extension method in Lemma 7.2 in [CRS10], where suitable trace and extended energies are compared in the unit ball: in a sense, since Proposition 2.3 here compares energies defined in the whole of the space, it can be viewed as a "global", or "renormalized", version of Lemma 7.2 in CRS10].
} 
Proposition 2.3. Let $n \geqslant 2$ and $s \in(0,1)$. For any $v \in W^{2, \infty}\left(\mathbb{R}^{n}\right)$ and any $\varphi \in C_{0}^{\infty}\left(\mathbb{R}^{n}\right)$, it holds that

$$
\begin{aligned}
+\infty & >\iint_{\mathbb{R}^{2 n}} \frac{|(v+\varphi)(x)-(v+\varphi)(y)|^{2}-|v(x)-v(y)|^{2}}{|x-y|^{n+2 s}} d x d y \\
& =\lim _{R \rightarrow+\infty} \int_{\mathcal{B}_{R}^{+}} z^{a}\left(\left|\nabla E_{v+\varphi}(x, z)\right|^{2}-\left|\nabla E_{v}(x, z)\right|^{2}\right) d x d z \\
& =\lim _{R \rightarrow+\infty} \inf _{\substack{\Phi \in C_{0}^{\infty}\left(\mathcal{B}_{R}\right) \\
\Phi(x, 0)=\varphi(x)}} \int_{\mathcal{B}_{R}^{+}} z^{a}\left(\left|\nabla\left(E_{v}+\Phi\right)(x, z)\right|^{2}-\left|\nabla E_{v}(x, z)\right|^{2}\right) d x d z .
\end{aligned}
$$

Proof. For concreteness, we suppose that the support of $\varphi$ lies in $B_{1}$. We take $\tau \in C_{0}^{\infty}\left(B_{2},[0,1]\right)$, with $\tau=1$ in $B_{1}$, and we let

$$
\tau_{k}(x):=\tau\left(\frac{x}{k}\right) \quad \text { and } \quad v_{k}(x):=\tau_{k}(x) v(x) .
$$

We also set $w_{k}:=v-v_{k}$. In this way, $w_{k}$ is bounded, uniformly Lipschitz and vanishes in $B_{k}$. In particular,

$$
\frac{\left|w_{k}(x)-w_{k}(y)\right||\varphi(x)-\varphi(y)|}{|x-y|^{n+2 s}} \leqslant \frac{C \min \{1,|x-y|\}|\varphi(x)-\varphi(y)|}{|x-y|^{n+2 s}} \in L^{1}\left(\mathbb{R}^{2 n}\right) .
$$

Furthermore, we have that

$$
\begin{aligned}
& \iint_{\mathbb{R}^{2 n}} \frac{|(v+\varphi)(x)-(v+\varphi)(y)|^{2}-|v(x)-v(y)|^{2}}{|x-y|^{n+2 s}} d x d y \\
& -\iint_{\mathbb{R}^{2 n}} \frac{\left|\left(v_{k}+\varphi\right)(x)-\left(v_{k}+\varphi\right)(y)\right|^{2}-\left|v_{k}(x)-v_{k}(y)\right|^{2}}{|x-y|^{n+2 s}} d x d y \\
= & 2 \iint_{\mathbb{R}^{2 n}} \frac{(v(x)-v(y))(\varphi(x)-\varphi(y))}{|x-y|^{n+2 s}} d x d y \\
& -2 \iint_{\mathbb{R}^{2 n}} \frac{\left(v_{k}(x)-v_{k}(y)\right)(\varphi(x)-\varphi(y))}{|x-y|^{n+2 s}} d x d y \\
= & 2 \iint_{\mathbb{R}^{2 n}} \frac{\left(w_{k}(x)-w_{k}(y)\right)(\varphi(x)-\varphi(y))}{|x-y|^{n+2 s}} d x d y .
\end{aligned}
$$

This, (2.8) and the Dominated Convergence Theorem give that

$$
\begin{aligned}
\lim _{k \rightarrow+\infty} & \iint_{\mathbb{R}^{2 n}} \frac{\left|\left(v_{k}+\varphi\right)(x)-\left(v_{k}+\varphi\right)(y)\right|^{2}-\left|v_{k}(x)-v_{k}(y)\right|^{2}}{|x-y|^{n+2 s}} d x d y \\
= & \iint_{\mathbb{R}^{2 n}} \frac{|(v+\varphi)(x)-(v+\varphi)(y)|^{2}-|v(x)-v(y)|^{2}}{|x-y|^{n+2 s}} d x d y .
\end{aligned}
$$

Also,

$$
\frac{|v(x)-v(y)||\varphi(x)-\varphi(y)|}{|x-y|^{n+2 s}} \leqslant \frac{C \min \{1,|x-y|\}|\varphi(x)-\varphi(y)|}{|x-y|^{n+2 s}} \in L^{1}\left(\mathbb{R}^{2 n}\right),
$$

and therefore

$$
\begin{aligned}
& \iint_{\mathbb{R}^{2 n}} \frac{|(v+\varphi)(x)-(v+\varphi)(y)|^{2}-|v(x)-v(y)|^{2}}{|x-y|^{n+2 s}} d x d y \\
& \quad=\iint_{\mathbb{R}^{2 n}} \frac{(v(x)-v(y))(\varphi(x)-\varphi(y))}{|x-y|^{n+2 s}} d x d y+\iint_{\mathbb{R}^{2 n}} \frac{|\varphi(x)-\varphi(y)|^{2}}{|x-y|^{n+2 s}} d x d y<+\infty .
\end{aligned}
$$


On the other hand, recalling (2.4),

$$
z^{a}|\nabla P(x, z)| \leqslant \frac{C z(|x|+z)}{\left(|x|^{2}+z^{2}\right)^{\frac{n+2 s+2}{2}}}+\frac{C}{\left(|x|^{2}+z^{2}\right)^{\frac{n+2 s}{2}}} \leqslant \frac{C}{\left(|x|^{2}+z^{2}\right)^{\frac{n+2 s}{2}}} .
$$

This implies that

$$
\begin{aligned}
& z^{a}\left|\nabla E_{\varphi}(x, z)\right|=\left|z^{a} \int_{\mathbb{R}^{n}} \nabla P(x-y, z) \varphi(y) d y\right| \\
& \quad \leqslant C \int_{B_{1}}|\nabla P(x-y, z)| d y \leqslant C \int_{B_{1}} \frac{d y}{\left(|x-y|^{2}+z^{2}\right)^{\frac{n+2 s}{2}}} .
\end{aligned}
$$

Hence, for any $x \in \mathbb{R}^{n} \backslash B_{2}$,

$$
z^{a}\left|\nabla E_{\varphi}(x, z)\right| \leqslant C \int_{B_{1}} \frac{d y}{\left(|x|^{2}+z^{2}\right)^{\frac{n+2 s}{2}}}=\frac{C}{\left(|x|^{2}+z^{2}\right)^{\frac{n+2 s}{2}}}
$$

and, if $x \in B_{2}$,

$$
z^{a}\left|\nabla E_{\varphi}(x, z)\right| \leqslant C \int_{B_{1}} \frac{d y}{\left(0+z^{2}\right)^{\frac{n+2 s}{2}}} \leqslant \frac{C}{z^{n+2 s}}
$$

We also notice that

$$
\begin{aligned}
& z^{a}\left|\nabla E_{\varphi}(x, z)\right| \leqslant z^{a}\left|\int_{B_{1}} P(y, z) \nabla \varphi(x-y) d y\right| \\
& \leqslant C z^{a} \int_{\mathbb{R}^{n}} P(y, z) d y \leqslant C z^{a} \in L^{1}\left(B_{2} \times(0,2)\right) .
\end{aligned}
$$

In addition, $\left\|E_{v_{k}}\right\|_{L^{\infty}\left(\mathbb{R}_{+}^{n+1}\right)} \leqslant\left\|v_{k}\right\|_{L^{\infty}\left(\mathbb{R}^{n}\right)}$; as a consequence of these observations, setting

$$
L_{R}:=\left(\partial B_{R}\right) \times(0, R) \text { and } U_{R}:=B_{R} \times\{R\},
$$

we have that

$$
\begin{gathered}
\lim _{R \rightarrow+\infty} \int_{L_{R} \cup U_{R}} z^{a}\left|E_{v_{k}}(x, z)\right|\left|\partial_{\nu} E_{\varphi}(x, z)\right| d \mathcal{H}^{n}(x, z) \leqslant \lim _{R \rightarrow+\infty} \int_{L_{R} \cup U_{R}} \frac{C d \mathcal{H}^{n}(x, z)}{\left(|x|^{2}+z^{2}\right)^{\frac{n+2 s}{2}}} \\
\leqslant \lim _{R \rightarrow+\infty} \int_{L_{R} \cup U_{R}} \frac{C d \mathcal{H}^{n}(x, z)}{R^{n+2 s}} \leqslant \lim _{R \rightarrow+\infty} \frac{C R^{n}}{R^{n+2 s}}=0,
\end{gathered}
$$

where $\partial_{\nu}$ denotes the external normal derivative to the boundary of $\mathcal{B}_{R}^{+}$.

Accordingly,

$$
\begin{aligned}
& \lim _{R \rightarrow+\infty} \int_{\mathcal{B}_{R}^{+}} z^{a}\left(\left|\nabla E_{v_{k}+\varphi}(x, z)\right|^{2}-\left|\nabla E_{v_{k}}(x, z)\right|^{2}\right) d x d z \\
= & \lim _{R \rightarrow+\infty} \int_{\mathcal{B}_{R}^{+}} z^{a}\left(\left|\nabla E_{\varphi}(x, z)\right|^{2}+2 \nabla E_{\varphi}(x, y) \cdot \nabla E_{v_{k}}(x, z)\right) d x d z \\
= & \lim _{R \rightarrow+\infty} \int_{\mathcal{B}_{R}^{+}} z^{a}\left|\nabla E_{\varphi}(x, z)\right|^{2}+2 \operatorname{div}\left(z^{a} E_{v_{k}}(x, y) \nabla E_{\varphi}(x, z)\right) d x d z \\
= & \lim _{R \rightarrow+\infty} \int_{\mathcal{B}_{R}^{+}} z^{a}\left|\nabla E_{\varphi}(x, z)\right|^{2} d x d z+2 \int_{\partial \mathcal{B}_{R}^{+}} z^{a} E_{v_{k}}(x, z) \partial_{\nu} E_{\varphi}(x, z) d \mathcal{H}^{n}(x, z) \\
= & \lim _{R \rightarrow+\infty} \int_{\mathcal{B}_{R}^{+}} z^{a}\left|\nabla E_{\varphi}(x, z)\right|^{2} d x d z+2 \int_{B_{R}} v_{k}(x)(-\Delta)^{s} \varphi(x) d x \\
= & \int_{\mathbb{R}_{+}^{n+1}} z^{a}\left|\nabla E_{\varphi}(x, z)\right|^{2} d x d z+2 \int_{\mathbb{R}^{n}} v_{k}(x)(-\Delta)^{s} \varphi(x) d x .
\end{aligned}
$$


Similarly,

$$
\begin{aligned}
& \lim _{R \rightarrow+\infty} \int_{\mathcal{B}_{R}^{+}} z^{a}\left(\left|\nabla E_{v+\varphi}(x, z)\right|^{2}-\left|\nabla E_{v}(x, z)\right|^{2}\right) d x d z \\
= & \int_{\mathbb{R}_{+}^{n+1}} z^{a}\left|\nabla E_{\varphi}(x, z)\right|^{2} d x d z+2 \int_{\mathbb{R}^{n}} v(x)(-\Delta)^{s} \varphi(x) d x .
\end{aligned}
$$

Since $\left|v_{k}(-\Delta)^{s} \varphi\right| \leqslant\left|v(-\Delta)^{s} \varphi\right| \leqslant \frac{C}{1+|x|^{n+2 s}} \in L^{1}\left(\mathbb{R}^{n}\right)$, we thus obtain that

$$
\begin{aligned}
& \lim _{k \rightarrow+\infty} \int_{\mathbb{R}_{+}^{n+1}} z^{a}\left(\left|\nabla E_{v_{k}+\varphi}(x, z)\right|^{2}-\left|\nabla E_{v_{k}}(x, z)\right|^{2}\right) d x d z \\
= & \lim _{k \rightarrow+\infty} \lim _{R \rightarrow+\infty} \int_{\mathcal{B}_{R}^{+}} z^{a}\left(\left|\nabla E_{v_{k}+\varphi}(x, z)\right|^{2}-\left|\nabla E_{v_{k}}(x, z)\right|^{2}\right) d x d z \\
= & \lim _{k \rightarrow+\infty}\left[\int_{\mathbb{R}_{+}^{n+1}} z^{a}\left|\nabla E_{\varphi}(x, z)\right|^{2} d x d z+2 \int_{\mathbb{R}^{n}} v_{k}(x)(-\Delta)^{s} \varphi(x) d x\right] \\
= & \int_{\mathbb{R}_{+}^{n+1}} z^{a}\left|\nabla E_{\varphi}(x, z)\right|^{2} d x d z+2 \int_{\mathbb{R}^{n}} v(x)(-\Delta)^{s} \varphi(x) d x \\
= & \lim _{R \rightarrow+\infty} \int_{\mathcal{B}_{R}^{+}} z^{a}\left(\left|\nabla E_{v+\varphi}(x, z)\right|^{2}-\left|\nabla E_{v}(x, z)\right|^{2}\right) d x d z .
\end{aligned}
$$

Moreover, from (2.6), we know that

$$
\begin{aligned}
& \iint_{\mathbb{R}^{2 n}} \frac{\left|\left(v_{k}+\varphi\right)(x)-\left(v_{k}+\varphi\right)(y)\right|^{2}-\left|v_{k}(x)-v_{k}(y)\right|^{2}}{|x-y|^{n+2 s}} d x d y \\
= & \lim _{R \rightarrow+\infty} \int_{\mathcal{B}_{R}^{+}} z^{a}\left(\left|\nabla E_{v_{k}+\varphi}(x, z)\right|^{2}-\left|\nabla E_{v_{k}}(x, z)\right|^{2}\right) d x d z .
\end{aligned}
$$

Putting together this with (2.9), (2.10) and (2.16), we conclude that

$$
\begin{aligned}
+\infty & >\iint_{\mathbb{R}^{2 n}} \frac{|(v+\varphi)(x)-(v+\varphi)(y)|^{2}-|v(x)-v(y)|^{2}}{|x-y|^{n+2 s}} d x d y \\
& =\lim _{R \rightarrow+\infty} \int_{\mathcal{B}_{R}^{+}} z^{a}\left(\left|\nabla E_{v+\varphi}(x, z)\right|^{2}-\left|\nabla E_{v}(x, z)\right|^{2}\right) d x d z .
\end{aligned}
$$

Therefore, to complete the proof of the desired claim, it remains to show that

$$
\begin{aligned}
& \lim _{R \rightarrow+\infty} \int_{\mathcal{B}_{R}^{+}} z^{a}\left(\left|\nabla E_{v+\varphi}(x, z)\right|^{2}-\left|\nabla E_{v}(x, z)\right|^{2}\right) d x d z \\
= & \lim _{R \rightarrow+\infty} \inf _{\substack{\Phi \in C_{0}^{\infty}\left(\mathcal{B}_{R}\right) \\
\Phi(x, 0)=\varphi(x)}} \int_{\mathcal{B}_{R}^{+}} z^{a}\left(\left|\nabla\left(E_{v}+\Phi\right)(x, z)\right|^{2}-\left|\nabla E_{v}(x, z)\right|^{2}\right) d x d z .
\end{aligned}
$$

To this end, we take $\theta \in C_{0}^{\infty}\left(\mathcal{B}_{1},[0,1]\right)$ with $\theta=1$ in $\mathcal{B}_{1 / 2}$ and we set $\theta_{R}(x, z):=\theta\left(\frac{x}{R}, \frac{z}{R}\right)$ and $\Phi_{R}:=$ $E_{\varphi} \theta_{R}$. We observe that

$$
\left|E_{\varphi}(x, z)\right| \leqslant \int_{B_{1}} \frac{C z^{2 s}}{\left(|x-y|^{2}+z^{2}\right)^{\frac{n+2 s}{2}}} d y
$$

and therefore, if $|x| \geqslant 2$,

$$
\left|E_{\varphi}(x, z)\right| \leqslant \frac{C z^{2 s}}{\left(|x|^{2}+z^{2}\right)^{\frac{n+2 s}{2}}} \leqslant \frac{C z^{2 s}}{\left(1+|x|^{2}+z^{2}\right)^{\frac{n+2 s}{2}}} .
$$


Similarly, if $|x| \leqslant 2$ and $z \geqslant 1$,

$$
\left|E_{\varphi}(x, z)\right| \leqslant \int_{B_{1}} \frac{C z^{2 s}}{\left(0+z^{2}\right)^{\frac{n+2 s}{2}}} d y \leqslant \frac{C z^{2 s}}{\left(1+|x|^{2}+z^{2}\right)^{\frac{n+2 s}{2}}},
$$

up to renaming $C$. Also, if $|x| \leqslant 2$ and $z \in(0,2)$,

$$
\left|E_{\varphi}(x, z)\right| \leqslant C \int_{\mathbb{R}^{n}} P(x-y, z) d y=C .
$$

In view of these estimates, we have that

$$
\begin{gathered}
\int_{\mathbb{R}_{+}^{n+1}} z^{a}\left|E_{\varphi}(x, z)\right|^{2} d x d z \leqslant C+\int_{\mathbb{R}_{+}^{n+1} \backslash \mathcal{B}_{2}} z^{a}\left|E_{\varphi}(x, z)\right|^{2} d x d z \\
\leqslant C+\int_{\mathbb{R}_{+}^{n+1} \backslash \mathcal{B}_{2}} \frac{C z^{1+2 s}}{\left(1+|x|^{2}+z^{2}\right)^{n+2 s}} d x d z \\
\leqslant C+\int_{\mathbb{R}_{+}^{n+1} \backslash \mathcal{B}_{2}} \frac{C}{\left(1+|x|^{2}+z^{2}\right)^{n+s-\frac{1}{2}}} d x d z \leqslant C,
\end{gathered}
$$

since $2 n+2 s-1>n+1$.

Similarly, recalling (2.11), (2.12) and (2.13),

$$
\begin{aligned}
& \int_{\mathbb{R}_{+}^{n+1}} z^{a}\left|\nabla E_{\varphi}(x, z)\right|^{2} d x d z \\
& \leqslant C+\int_{\left\{(x, z): x \in B_{2}, z>1\right\}} z^{-a}\left(z^{a}\left|\nabla E_{\varphi}(x, z)\right|\right)^{2} d x d z+\int_{\left\{(x, z): x \in \mathbb{R}^{n} \backslash B_{2}, z \in(0,1]\right\}} z^{-a}\left(z^{a}\left|\nabla E_{\varphi}(x, z)\right|\right)^{2} d x d z \\
& \quad+\int_{\left\{(x, z): x \in \mathbb{R}^{n} \backslash B_{2}, z>1\right\}} z^{-a}\left(z^{a}\left|\nabla E_{\varphi}(x, z)\right|\right)^{2} d x d z \\
& \quad+\quad \frac{C}{\int_{\left\{(x, z): x \in B_{2}, z>1\right\}}} \frac{C}{z^{2 n+1+2 s}} d x d z+\int_{\left\{(x, z): x \in \mathbb{R}^{n} \backslash B_{2}, z \in(0,1]\right\}} \frac{C}{z^{1-2 s}\left(|x|^{2}+0\right)^{n+2 s}} d x d z \\
& \quad+\int_{\left\{(x, z): x \in \mathbb{R}^{n} \backslash B_{2}, z>1\right\}} \frac{C}{\left(|x|^{2}+z^{2}\right)^{n+2 s}} d x d z
\end{aligned}
$$

and therefore

$$
\int_{\mathbb{R}_{+}^{n+1} \backslash \mathcal{B}_{R / 2}} z^{a}\left|\nabla E_{\varphi}(x, z)\right|^{2} d x d z \leqslant \delta(R),
$$

with $\delta(R)$ infinitesimal as $R \rightarrow+\infty$.

In addition,

$$
\begin{aligned}
\left.|| \nabla \Phi_{R}\right|^{2}-\left|\nabla E_{\varphi}\right|^{2} \mid & \leqslant E_{\varphi}^{2}\left|\nabla \theta_{R}\right|^{2}+\left|\nabla E_{\varphi}\right|^{2}\left|1-\theta_{R}^{2}\right|+2\left|E_{\varphi}\right|\left|\theta_{R}\right|\left|\nabla E_{\varphi}\right|\left|\nabla \theta_{R}\right| \\
& \leqslant \frac{C E_{\varphi}^{2}}{R^{2}}+C\left|\nabla E_{\varphi}\right|^{2} \chi_{\mathbb{R}_{+}^{n+1} \backslash \mathcal{B}_{R / 2}} .
\end{aligned}
$$


Therefore, in light of (2.18) and (2.19),

$$
\begin{aligned}
& \left.\left|\int_{\mathbb{R}_{+}^{n+1}} z^{a}\right| \nabla \Phi_{R}(x, z)\right|^{2} d x d z-\int_{\mathbb{R}_{+}^{n+1}} z^{a}\left|\nabla E_{\varphi}(x, z)\right|^{2} d x d z \mid \\
\leqslant & \frac{C}{R^{2}} \int_{\mathbb{R}_{+}^{n+1}} z^{a}\left|E_{\varphi}(x, z)\right|^{2} d x d z+C \int_{\mathbb{R}_{+}^{n+1} \backslash \mathcal{B}_{R / 2}} z^{a}\left|\nabla E_{\varphi}(x, z)\right|^{2} d x d z \\
\leqslant & \frac{C}{R^{2}}+\delta(R) .
\end{aligned}
$$

Consequently, we find that

$$
\begin{aligned}
& \lim _{R \rightarrow+\infty} \inf _{\substack{\Phi \in C_{0}^{\infty}\left(\mathcal{B}_{R}\right) \\
\Phi(x, 0)=\varphi(x)}} \int_{\mathcal{B}_{R}^{+}} z^{a}\left(\left|\nabla\left(E_{v}+\Phi\right)(x, z)\right|^{2}-\left|\nabla E_{v}(x, z)\right|^{2}\right) d x d z \\
\leqslant & \lim _{R \rightarrow+\infty} \int_{\mathcal{B}_{R}^{+}} z^{a}\left(\left|\nabla\left(E_{v}+\Phi_{R}\right)(x, z)\right|^{2}-\left|\nabla E_{v}(x, z)\right|^{2}\right) d x d z \\
= & \lim _{R \rightarrow+\infty} \int_{\mathcal{B}_{R}^{+}} z^{a}\left(\left|\nabla \Phi_{R}(x, z)\right|^{2}+2 \nabla E_{v}(x, z) \cdot \nabla \Phi_{R}(x, z)\right) d x d z \\
\leqslant & \lim _{R \rightarrow+\infty}\left[\frac{C}{R^{2}}+\delta(R)+\int_{\mathcal{B}_{R}^{+}} z^{a}\left(\left|\nabla E_{\varphi}(x, z)\right|^{2}+2 \nabla E_{v}(x, z) \cdot \nabla E_{\varphi}(x, z)\right) d x d z\right. \\
+ & \left.\int_{\mathcal{B}_{R}^{+}} z^{a} \nabla E_{v}(x, z) \cdot \nabla\left(\Phi \Phi_{R}(x, z)-E_{\varphi}(x, z)\right) d x d z\right] \\
\leqslant & \lim _{R \rightarrow+\infty}\left[\frac{C}{R^{2}}+\delta(R)+\int_{\mathcal{B}_{R}^{+}} z^{a}\left(\left|\nabla E_{\varphi}(x, z)\right|^{2}+2 \nabla E_{v}(x, z) \cdot \nabla E_{\varphi}(x, z)\right) d x d z\right. \\
+ & \left.\sup _{\substack{\Phi \in C_{0}^{\infty}\left(\mathcal{B}_{R}\right) \\
\Phi(x, 0)=\varphi(x)}} \int_{\mathcal{B}_{R}^{+}} z^{a} \nabla E_{v}(x, z) \cdot \nabla\left(\Phi(x, z)-E_{\varphi}(x, z)\right) d x d z\right] .
\end{aligned}
$$

Now we claim that

$$
\lim _{R \rightarrow+\infty} \sup _{\substack{\Phi \in C_{0}^{\infty}\left(\mathcal{B}_{R}\right) \\ \Phi(x, 0)=\varphi(x)}}\left|\int_{\mathcal{B}_{R}^{+}} z^{a} \nabla E_{v}(x, z) \cdot \nabla\left(\Phi(x, z)-E_{\varphi}(x, z)\right) d x d z\right|=0 .
$$

To check this, we recall the notation in (2.14) and observe that

$$
\begin{aligned}
& \lim _{R \rightarrow+\infty} \sup _{\substack{\Phi \in C_{0}^{\infty}\left(\mathcal{B}_{R}\right) \\
\Phi(x, 0)=\varphi(x)}}\left|\int_{\mathcal{B}_{R}^{+}} z^{a} \nabla E_{v}(x, z) \cdot \nabla\left(\Phi(x, z)-E_{\varphi}(x, z)\right) d x d z\right| \\
& =\lim _{R \rightarrow+\infty} \sup _{\substack{\Phi \in C_{0}^{\infty}\left(\mathcal{B}_{R}\right) \\
\Phi(x, 0)=\varphi(x)}}\left|\int_{\mathcal{B}_{R}^{+}} \operatorname{div}\left(z^{a} \Phi(x, z) \nabla E_{v}(x, z)\right) d x d z-\int_{\mathcal{B}_{R}^{+}} \operatorname{div}\left(z^{a} E_{v}(x, z) \nabla E_{\varphi}(x, z)\right) d x d z\right| \\
& =\lim _{R \rightarrow+\infty} \mid \int_{B_{R}} \varphi(x)(-\Delta)^{s} v(x) d x-\int_{B_{R}} v(x)(-\Delta)^{s} \varphi(x) d x \\
& \quad-\int_{L_{R} \cup U_{R}} z^{a} E_{v}(x, z) \partial_{\nu} E_{\varphi}(x, z) d \mathcal{H}^{n}(x, z) \mid .
\end{aligned}
$$


Also, as in (2.15), we have that

$$
\lim _{R \rightarrow+\infty} \int_{L_{R} \cup U_{R}} z^{a}\left|E_{v}(x, z)\right|\left|\partial_{\nu} E_{\varphi}(x, z)\right| d \mathcal{H}^{n}(x, z)=0 .
$$

Hence, fixing $k$ and recalling the notation in (2.7), we derive from (2.22) that

$$
\begin{aligned}
& \lim _{R \rightarrow+\infty} \sup _{\substack{\Phi \in C_{0}^{\infty}\left(\mathcal{B}_{R}\right) \\
\Phi(x, 0)=\varphi(x)}}\left|\int_{\mathcal{B}_{R}^{+}} z^{a} \nabla E_{v}(x, z) \cdot \nabla\left(\Phi(x, z)-E_{\varphi}(x, z)\right) d x d z\right| \\
= & \left|\int_{\mathbb{R}^{n}} \varphi(x)(-\Delta)^{s} v(x) d x-\int_{\mathbb{R}^{n}} v(x)(-\Delta)^{s} \varphi(x) d x\right| \\
= & \mid \int_{\mathbb{R}^{n}} \varphi(x)(-\Delta)^{s} v_{k}(x) d x-\int_{\mathbb{R}^{n}} v_{k}(x)(-\Delta)^{s} \varphi(x) d x \\
= & \left|\int_{\mathbb{R}^{n}} \varphi(x)(-\Delta)^{s} w_{k}(x) d x-\int_{\mathbb{R}^{n}} \varphi(x)(-\Delta)^{s} w_{k}(x) d x-\int_{\mathbb{R}^{n}} w_{k}(x)(-\Delta)^{s} \varphi(x) d x\right|
\end{aligned}
$$

Since $\left|(-\Delta)^{s} \varphi(x)\right| \leqslant \frac{C}{1+|x|^{n+2 s}} \in L^{1}\left(\mathbb{R}^{n}\right)$, we can take the limit as $k \rightarrow+\infty$ and use the Dominated Convergence Theorem, to obtain that

$$
\begin{aligned}
& \lim _{R \rightarrow+\infty} \sup _{\substack{\Phi \in C_{0}^{\infty}\left(\mathcal{B}_{R}\right) \\
\Phi(x, 0)=\varphi(x)}}\left|\int_{\mathcal{B}_{R}^{+}} z^{a} \nabla E_{v}(x, z) \cdot \nabla\left(\Phi(x, z)-E_{\varphi}(x, z)\right) d x d z\right| \\
= & \lim _{k \rightarrow+\infty}\left|\int_{\mathbb{R}^{n}} \varphi(x)(-\Delta)^{s} w_{k}(x) d x-\int_{\mathbb{R}^{n}} w_{k}(x)(-\Delta)^{s} \varphi(x) d x\right| \\
= & \lim _{k \rightarrow+\infty}\left|\int_{\mathbb{R}^{n}} \varphi(x)(-\Delta)^{s} w_{k}(x) d x\right| \\
= & \lim _{k \rightarrow+\infty}\left|\int_{\mathbb{R}^{n}} \varphi(x)(-\Delta)^{s} v(x) d x-\int_{\mathbb{R}^{n}} \varphi(x)(-\Delta)^{s}\left(\tau_{k} v\right)(x) d x\right| \\
= & \mid \int_{\mathbb{R}^{n}} \varphi(x)(-\Delta)^{s} v(x) d x-\lim _{k \rightarrow+\infty}\left[\int_{\mathbb{R}^{n}} \tau_{k}(x) \varphi(x)(-\Delta)^{s} v(x) d x\right. \\
& \left.+\int_{\mathbb{R}^{n}} v(x) \varphi(x)(-\Delta)^{s} \tau_{k}(x) d x+\int_{\mathbb{R}^{n}} \varphi(x) B\left(\tau_{k}, v\right)(x) d x\right] \mid \\
= & \lim _{k \rightarrow+\infty}\left|\frac{1}{k^{2 s}} \int_{B_{1}} v(x) \varphi(x)(-\Delta)^{s} \tau\left(\frac{x}{k}\right) d x+\int_{\mathbb{R}^{n}} \varphi(x) B\left(\tau_{k}, v\right)(x) d x\right| \\
\leqslant & \lim _{k \rightarrow+\infty}\left[\frac{C}{k^{2 s}} \int_{B_{1}}|v(x)||\varphi(x)| d x+\int_{\mathbb{R}^{n}}|\varphi(x)|\left|B\left(\tau_{k}, v\right)(x)\right| d x\right] \\
= & \lim _{k \rightarrow+\infty} \int_{B_{1}}|\varphi(x)|\left|B\left(\tau_{k}, v\right)(x)\right| d x,
\end{aligned}
$$

where

$$
B(f, g):=c \int_{\mathbb{R}^{n}} \frac{(f(x)-f(y))(g(x)-g(y))}{|x-y|^{n+2 s}} d y,
$$

for some $c>0$, see e.g. page 636 in [BPSV14] for such bilinear form. 
Notice now that

$$
\begin{aligned}
\left|B\left(\tau_{k}, v\right)(x)\right| & \leqslant C \int_{\mathbb{R}^{n}} \frac{\min \left\{1, \frac{|x-y|}{k}\right\} \min \{1,|x-y|\}}{|x-y|^{n+2 s}} d y \\
& \leqslant \frac{C}{k} \int_{B_{1}(x)} \frac{|x-y|^{2}}{|x-y|^{n+2 s}} d y+\frac{C}{k} \int_{B_{k}(x) \backslash B_{1}(x)} \frac{|x-y|}{|x-y|^{n+2 s}} d y+C \int_{\mathbb{R}^{n} \backslash B_{k}(x)} \frac{d y}{|x-y|^{n+2 s}} \\
& \leqslant \frac{C}{k}+\frac{C}{k^{2 s}} .
\end{aligned}
$$

We plug this information into (2.23) and we obtain (2.21), as desired.

Now, we insert (2.21) into (2.20) and we conclude that

$$
\begin{aligned}
& \lim _{R \rightarrow+\infty} \inf _{\substack{\Phi \in C_{0}^{\infty}\left(\mathcal{B}_{R}\right) \\
\Phi(x, 0)=\varphi(x)}} \int_{\mathcal{B}_{R}^{+}} z^{a}\left(\left|\nabla\left(E_{v}+\Phi\right)(x, z)\right|^{2}-\left|\nabla E_{v}(x, z)\right|^{2}\right) d x d z \\
& \quad \leqslant \lim _{R \rightarrow+\infty} \int_{\mathcal{B}_{R}^{+}} z^{a}\left(\left|\nabla E_{\varphi}(x, z)\right|^{2}+2 \nabla E_{v}(x, z) \cdot \nabla E_{\varphi}(x, z)\right) d x d z \\
& \quad=\lim _{R \rightarrow+\infty} \int_{\mathcal{B}_{R}^{+}} z^{a}\left(\left|\nabla E_{v+\varphi}(x, z)\right|^{2}-\left|\nabla E_{v}(x, z)\right|^{2}\right) d x d z
\end{aligned}
$$

Hence, to prove (2.17), it remains to show that

$$
\begin{aligned}
& \lim _{R \rightarrow+\infty} \int_{\mathcal{B}_{R}^{+}} z^{a}\left(\left|\nabla E_{v+\varphi}(x, z)\right|^{2}-\left|\nabla E_{v}(x, z)\right|^{2}\right) d x d z \\
\leqslant & \lim _{R \rightarrow+\infty} \inf _{\substack{\Phi \in C_{0}^{\infty}\left(\mathcal{B}_{R}\right) \\
\Phi(x, 0)=\varphi(x)}} \int_{\mathcal{B}_{R}^{+}} z^{a}\left(\left|\nabla\left(E_{v}+\Phi\right)(x, z)\right|^{2}-\left|\nabla E_{v}(x, z)\right|^{2}\right) d x d z .
\end{aligned}
$$

For this, we fix $\Psi \in C_{0}^{\infty}\left(\mathcal{B}_{R}\right)$ with $\Psi(x, 0)=\varphi(x)$ and we use again (2.21) to see that

$$
\begin{aligned}
& \int_{\mathcal{B}_{R}^{+}} z^{a}\left(\left|\nabla\left(E_{v}+\Psi\right)(x, z)\right|^{2}-\left|\nabla E_{v}(x, z)\right|^{2}\right) d x d z \\
= & \int_{\mathcal{B}_{R}^{+}} z^{a}\left(|\nabla \Psi(x, z)|^{2}+2 z^{a} \nabla \Psi(x, z) \cdot \nabla E_{v}(x, z)\right) d x d z \\
= & \int_{\mathcal{B}_{R}^{+}} z^{a}\left(|\nabla \Psi(x, z)|^{2}+2 z^{a} \nabla E_{\varphi}(x, z) \cdot \nabla E_{v}(x, z)\right) d x d z \\
\geqslant & \int_{\mathcal{B}_{R}^{+}} z^{a}\left(|\nabla \Psi(x, z)|^{2}+2 z^{a} \nabla E_{\varphi}(x, z) \cdot \nabla E_{v}(x, z)\right) d x d z \\
= & \int_{\mathcal{B}_{R}^{+}} z^{a} \nabla E_{v}(x, z) \cdot \nabla\left(\Psi(x, z)-E_{\varphi}(x, z)\right) d x d z \\
& -2 \underset{\substack{\Phi \in C_{0}^{\infty}\left(\mathcal{B}_{R}\right) \\
\Phi(x, 0)=\varphi(x)}}{\sup _{\mathcal{B}_{R}}} \mid \int_{\mathcal{B}_{R}^{+}} z^{a} \nabla E_{v}(x, z) \cdot \nabla\left(\left.\Phi(x, z)\right|^{2}+2 z^{a} \nabla E_{\varphi}(x, z) \cdot \nabla E_{v}(x, z)\right) d x d z-\mu(R),
\end{aligned}
$$

with $\mu(R)$ independent of $\Psi$ and infinitesimal as $R \rightarrow+\infty$.

Now we take $\Psi_{*} \in C_{0}^{\infty}\left(\mathcal{B}_{R}\right)$ such that $\Psi_{*}(x, 0)=\varphi(x)$ that minimizes the functional $\Psi \mapsto$ $\int_{\mathcal{B}_{R}^{+}} z^{a}|\nabla \Psi(x, z)|^{2} d x d z$ in such class. Then, using the variational equation for minimizers inside $\mathcal{B}_{R}^{+}$ 
and the fact that $\Psi_{*}(x, 0)=E_{\varphi}(x, 0)$, we see that

$$
\begin{aligned}
& \int_{\mathcal{B}_{R}^{+}} z^{a}\left(|\nabla \Psi(x, z)|^{2}-\left|\nabla E_{\varphi}(x, z)\right|^{2}\right) d x d z \\
\geqslant & \int_{\mathcal{B}_{R}^{+}} z^{a}\left(\left|\nabla \Psi_{*}(x, z)\right|^{2}-\left|\nabla E_{\varphi}(x, z)\right|^{2}\right) d x d z \\
= & \int_{\mathcal{B}_{R}^{+}} z^{a} \nabla\left(\Psi_{*}-E_{\varphi}\right)(x, z) \cdot \nabla\left(\Psi_{*}+E_{\varphi}\right)(x, z) d x d z \\
= & \int_{\mathcal{B}_{R}^{+}} \operatorname{div}\left(z^{a}\left(\Psi_{*}-E_{\varphi}\right)(x, z) \nabla\left(\Psi_{*}+E_{\varphi}\right)(x, z)\right) d x d z \\
= & \int_{L_{R} \cup U_{R}} z^{a}\left(\Psi_{*}-E_{\varphi}\right)(x, z) \partial_{\nu}\left(\Psi_{*}+E_{\varphi}\right)(x, z) d \mathcal{H}^{n}(x, z) \\
= & -\int_{L_{R} \cup U_{R}} z^{a} E_{\varphi}(x, z) \partial_{\nu} E_{\varphi}(x, z) d \mathcal{H}^{n}(x, z) .
\end{aligned}
$$

Therefore, recalling (2.11) and (2.12), we conclude that

$$
\int_{\mathcal{B}_{R}^{+}} z^{a}\left(|\nabla \Psi(x, z)|^{2}-\left|\nabla E_{\varphi}(x, z)\right|^{2}\right) d x d z \geqslant-\int_{L_{R} \cup U_{R}} \frac{C}{R^{n+2 s}} d \mathcal{H}^{n}(x, z) \geqslant-\frac{C}{R^{2 s}} .
$$

From this and (2.25) we thus obtain that

$$
\begin{aligned}
& \int_{\mathcal{B}_{R}^{+}} z^{a}\left(\left|\nabla\left(E_{v}+\Psi\right)(x, z)\right|^{2}-\left|\nabla E_{v}(x, z)\right|^{2}\right) d x d z \\
\geqslant & \int_{\mathcal{B}_{R}^{+}} z^{a}\left(\left|\nabla E_{\varphi}(x, z)\right|^{2}+2 z^{a} \nabla E_{\varphi}(x, z) \cdot \nabla E_{v}(x, z)\right) d x d z-\mu(R)-\frac{C}{R^{2 s}} \\
= & \int_{\mathcal{B}_{R}^{+}} z^{a}\left(\left|\nabla E_{\varphi+v}(x, z)\right|^{2}-\left|\nabla E_{v}(x, z)\right|^{2}\right) d x d z-\mu(R)-\frac{C}{R^{2 s}} .
\end{aligned}
$$

Since this is valid for any $\Psi \in C_{0}^{\infty}\left(\mathcal{B}_{R}\right)$ with $\Psi(x, 0)=\varphi(x)$, we conclude that

$$
\begin{aligned}
& \inf _{\substack{\Phi \in C_{0}^{\infty}\left(\mathcal{B}_{R}\right) \\
\Phi(x, 0)=\varphi(x)}} \int_{\mathcal{B}_{R}^{+}} z^{a}\left(\left|\nabla\left(E_{v}+\Phi\right)(x, z)\right|^{2}-\left|\nabla E_{v}(x, z)\right|^{2}\right) d x d z \\
\geqslant & \int_{\mathcal{B}_{R}^{+}} z^{a}\left(\left|\nabla E_{v+\varphi}(x, z)\right|^{2}-\left|\nabla E_{v}(x, z)\right|^{2}\right) d x d z-\mu(R)-\frac{C}{R^{2 s}} .
\end{aligned}
$$

By taking the limit as $R \rightarrow+\infty$, we obtain (2.24), as desired, and so we have completed the proof of Proposition 2.3.

In view of Proposition 2.3, we can now relate the original and the extended energy functionals, according to the following result:

Corollary 2.4. Let $n \geqslant 2$ and $s \in(0,1)$. For any $v \in W^{2, \infty}\left(\mathbb{R}^{n}\right)$ it holds that

$$
\inf _{\substack{R>0 \\ \Phi \in C_{0}^{\infty}\left(\mathcal{B}_{R}\right)}} \mathcal{E}_{\mathcal{B}_{R}}\left(E_{v}+\Phi\right)-\mathcal{E}_{\mathcal{B}_{R}}\left(E_{v}\right)=\inf _{\substack{R>0 \\ \varphi \in C_{0}^{\infty}\left(B_{R}\right)}} \mathcal{F}_{B_{R}}(v+\varphi)-\mathcal{F}_{B_{R}}(v)
$$


Proof. Notice that, given $\varphi \in C_{0}^{\infty}\left(\mathbb{R}^{n}\right)$,

$$
\begin{aligned}
& \inf _{\substack{R>0 \\
\Phi \in C_{0}^{\infty}\left(\mathcal{B}_{R}\right) \\
\Phi(x, 0)=\varphi(x)}} \mathcal{E}_{\mathcal{B}_{R}}\left(E_{v}+\Phi\right)-\mathcal{E}_{\mathcal{B}_{R}}\left(E_{v}\right)
\end{aligned}
$$

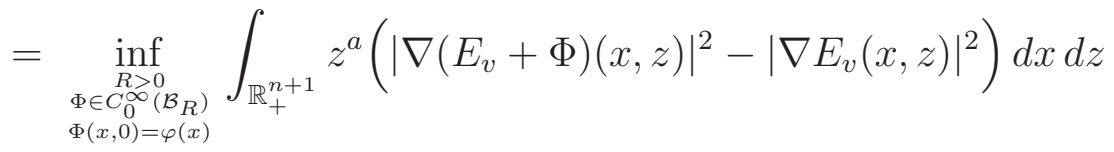

$$
\begin{aligned}
& +\int_{\mathbb{R}^{n}}(F(v(x)+\varphi(x))-F(v(x))) d x \\
& =\lim _{R \rightarrow+\infty} \inf _{\substack{\Phi \in C_{0}^{\infty}\left(\mathcal{B}_{R}\right) \\
\Phi(x, 0)=\varphi(x)}} \int_{\mathbb{R}_{+}^{n+1}} z^{a}\left(\left|\nabla\left(E_{v}+\Phi\right)(x, z)\right|^{2}-\left|\nabla E_{v}(x, z)\right|^{2}\right) d x d z \\
& +\int_{\mathbb{R}^{n}}(F(v(x)+\varphi(x))-F(v(x))) d x \\
& =\iint_{\mathbb{R}^{2 n}} \frac{|(v+\varphi)(x)-(v+\varphi)(y)|^{2}-|v(x)-v(y)|^{2}}{|x-y|^{n+2 s}} d x d y \\
& +\int_{\mathbb{R}^{n}}(F(v(x)+\varphi(x))-F(v(x))) d x \\
& =\mathcal{F}_{B_{R}}(v+\varphi)-\mathcal{F}_{B_{R}}(v),
\end{aligned}
$$

thanks to Proposition 2.3. Since the identity in (2.27) is valid for any $\varphi \in C_{0}^{\infty}\left(\mathbb{R}^{n}\right)$ (i.e. for any $R>0$ and any $\left.\varphi \in C_{0}^{\infty}\left(B_{R}\right)\right)$, taking the infimum in such class we obtain (2.26), as desired.

Due to Corollary 2.4, the following equivalence result for minimizers holds true:

Proposition 2.5. $E_{v}$ is an extended local minimizer according to Definition 2.2 if and only if $v$ is a local minimizer according to Definition 2.1.

Proof. We observe that $E_{v}$ is an extended local minimizer according to Definition 2.2 if and only if the first term in (2.26) is nonnegative; on the other hand, $v$ is a local minimizer according to Definition 2.1 if and only if the last term in (2.26) is nonnegative; since the two terms in (2.26) are equal, the desired result is established.

In view of Proposition 2.5 (see also Lemma 6.1 in [CS15]), it is natural to say that $u$ is a stable solution of (2.1) if the second derivative of the associated energy functional is nonnegative, according to the following setting:

Definition 2.6. Let $u$ be a solution of (2.1) in $\mathbb{R}^{n}$. We say that $u$ is stable if

$$
\int_{\mathbb{R}_{+}^{n+1}} z^{a}|\nabla \zeta(x, z)|^{2} d x d z+\int_{\mathbb{R}^{n}} F^{\prime \prime}(u(x)) \zeta^{2}(x, 0) d x \geqslant 0
$$

for any $\zeta \in C_{0}^{\infty}\left(\mathbb{R}^{n+1}\right)$.

\section{VARIational CLASSiFication OF 1D SOlUtions}

The goal of this section is to establish the following result:

Lemma 3.1. Let $s \in(0,1)$ and $v \in C^{2}(\mathbb{R},[-1,1])$ be a stable solution of $(-\Delta)^{s} v=f(v)$ in $\mathbb{R}$. Assume also that $\dot{v} \geqslant 0$. Then $v$ is a local minimizer.

Proof. The monotonicity of $v$ implies that the following limits exist:

$$
\underline{\ell}:=\lim _{t \rightarrow-\infty} v(t) \leqslant \lim _{t \rightarrow+\infty} v(t)=: \bar{\ell} .
$$


We also consider the sequence of functions $v_{k}(t):=v(t+k)$. By the Theorem of Ascoli, up to a subsequence we know that $v_{k}$ converges to $\bar{\ell}$ in $C_{\mathrm{loc}}^{2}(\mathbb{R})$, and so, passing the equation to the limit, we conclude that $f(\bar{\ell})=0$. Similarly, one sees that $f(\underline{\ell})=0$. As a consequence,

$$
\underline{\ell}, \bar{\ell} \in\{-1,0,1\} \text {. }
$$

Now, we claim that

$v$ is not identically zero.

The proof is by contradiction: if $v$ is identically zero, we take $\psi \in C_{0}^{\infty}\left(\mathbb{R}^{2}\right)$ and, for $\varepsilon>0$, we let $\psi_{\varepsilon}(x, z):=\psi(\varepsilon x, \varepsilon z)$. The stability inequality for $\psi_{\varepsilon}$ gives that

$$
\begin{aligned}
0 & \leqslant \int_{\mathbb{R}_{+}^{2}} z^{a}\left|\nabla \psi_{\varepsilon}(x, z)\right|^{2} d x d z+\int_{\mathbb{R}} F^{\prime \prime}(v(x)) \psi_{\varepsilon}^{2}(x, 0) d x \\
& =\varepsilon^{2} \int_{\mathbb{R}_{+}^{2}} z^{a}|\nabla \psi(\varepsilon x, \varepsilon z)|^{2} d x d z+\int_{\mathbb{R}} F^{\prime \prime}(v(x)) \psi^{2}(\varepsilon x, 0) d x \\
& =\varepsilon^{-a} \int_{\mathbb{R}_{+}^{2}} Z^{a}|\nabla \psi(X, Z)|^{2} d X d Z-\varepsilon^{-1} \int_{\mathbb{R}} f^{\prime}(0) \psi^{2}(X, 0) d X \\
& =C_{1} \varepsilon^{2 s-1}-C_{2} \varepsilon^{-1} f^{\prime}(0),
\end{aligned}
$$

for some $C_{1}, C_{2}>0$. From this, one obtains that

$$
f^{\prime}(0) \leqslant \lim _{\varepsilon \searrow 0} \frac{C_{1} \varepsilon^{2 s}}{C_{2}}=0 .
$$

This is a contradiction, since $f^{\prime}(0)>0$ and thus (3.2) is proved.

To complete the proof of Lemma 3.1, we now distinguish two cases, either $v$ is constant or not. If $v$ is constant, then it is either identically -1 or identically 1 , due to (3.2), and this implies the desired result.

So, we can now focus on the case in which $v$ is not constant. Then, $\underline{\ell}<\bar{\ell}$. So, from (3.1), we have that $v$ is a transition layer connecting:

(1) either -1 to 0 ,

(2) or 0 to 1 ,

(3) or -1 to 1 .

In view of Theorem 2.2(i) in [CS14], the first two cases cannot occur and therefore

$$
\lim _{t \rightarrow \pm \infty} v(t)= \pm 1 \text {. }
$$

Since the proof of this fact relies on the theory of layer solutions, we provide the details of the argument that we used. We argue for a contradiction and we suppose that

$$
\begin{aligned}
& \text { either } \lim _{t \rightarrow-\infty} v(t)=-1 \text { and } \lim _{t \rightarrow+\infty} v(t)=0 \\
& \text { or } \lim _{t \rightarrow-\infty} v(t)=0 \text { and } \lim _{t \rightarrow+\infty} v(t)=1 .
\end{aligned}
$$

By maximum principle, we know that $\dot{v}>0$. Then, if we set either $\tilde{v}(t):=2 v(t)+1$ (if the case in (3.4) holds true) or $\tilde{v}(t):=2 v(t)-1$ (if (3.5) holds true), we have that the derivative of $\tilde{v}$ is strictly positive and

$$
\lim _{t \rightarrow \pm \infty} \tilde{v}(t)= \pm 1
$$

In addition,

$$
(-\Delta)^{s} \tilde{v}(t)=2(-\Delta)^{s} v(t)=2 f(v(t))=2 f\left(\frac{\tilde{v}(t) \mp 1}{2}\right)=-G^{\prime}(\tilde{v}(t))
$$


with

$$
G(r):=-2 \int_{0}^{r} f\left(\frac{\tau \mp 1}{2}\right) d \tau=-4 \int_{\mp 1 / 2}^{(r \mp 1) / 2} f(\sigma) d \sigma .
$$

This and (3.6) give that we are in the setting of Theorem 2.2(i) in CS14. In particular, from formula (2.8) in CS14 we know that

$$
0=G(-1)-G(1)=4 \int_{\mp 1 / 2}^{(1 \mp 1) / 2} f(\sigma) d \sigma-4 \int_{\mp 1 / 2}^{(-1 \mp 1) / 2} f(\sigma) d \sigma=4 \int_{(-1 \mp 1) / 2}^{(1 \mp 1) / 2} f(\sigma) d \sigma
$$

hence

$$
\text { either } \quad \int_{-1}^{0} f(\sigma) d \sigma=0 \quad \text { or } \quad \int_{0}^{1} f(\sigma) d \sigma=0
$$

This is in contradiction with (2.2) and so it proves (3.3).

Hence, necessarily $v$ is a transition layer connecting -1 to 1 and so it is minimal due to the sliding method (see e.g. the proof of Lemma 9.1 in [VSS06]).

\section{Classification of the profiles at infinity}

In this section, we consider the two profiles of a given solution at infinity. Namely, if $s \in(0,1)$ and $u \in C^{2}\left(\mathbb{R}^{n},[-1,1]\right)$ is a solution of $(-\Delta)^{s} u=f(u)$ in $\mathbb{R}^{n}$, with $\partial_{x_{n}} u>0$ in $\mathbb{R}^{n}$, we set

$$
\underline{u}\left(x^{\prime}\right):=\lim _{x_{n} \rightarrow-\infty} u\left(x^{\prime}, x_{n}\right) \text { and } \bar{u}\left(x^{\prime}\right):=\lim _{x_{n} \rightarrow+\infty} u\left(x^{\prime}, x_{n}\right) .
$$

In this setting, we have:

Lemma 4.1. Assume that $n=3$. Then, both $\underline{u}$ and $\bar{u}$ are $1 D$ and local minimizers.

Proof. By passing the equation to the limit, we have that

$$
\text { both } \underline{u} \text { and } \bar{u} \text { are stable solutions in } \mathbb{R}^{n-1}=\mathbb{R}^{2} \text {. }
$$

The proof of (4.1) is based on a general argument (see e.g. [FSV08]), given in details here for the sake of completeness. Let $\xi \in C_{0}^{\infty}\left(\mathbb{R}^{n}\right)$ and $\eta \in C_{0}^{\infty}((-1,1))$, with $\eta(0)=1$. Given $\varepsilon>0$, we set $\xi_{\varepsilon}(x, z)=\xi_{\varepsilon}\left(x^{\prime}, x_{n}, z\right):=\xi\left(x^{\prime}, z\right) \eta\left(\varepsilon x_{n}\right)$. Notice that $\xi_{\varepsilon} \in C_{0}^{\infty}\left(\mathbb{R}^{n+1}\right)$, therefore by the stability of $u$ and the translation invariance we have that

$$
\begin{aligned}
0 \leqslant & \lim _{t \rightarrow+\infty} C \int_{\mathbb{R}_{+}^{n+1}} z^{a}\left|\nabla \xi_{\varepsilon}(x, z)\right|^{2} d x d z+\int_{\mathbb{R}^{n}} F^{\prime \prime}\left(u\left(x^{\prime}, x_{n}+t\right)\right) \xi_{\varepsilon}(x, 0) d x \\
= & C \int_{\mathbb{R}_{+}^{n+1}} z^{a}\left|\nabla \xi_{\varepsilon}(x, z)\right|^{2} d x d z+\int_{\mathbb{R}^{n}} F^{\prime \prime}\left(\bar{u}\left(x^{\prime}\right)\right) \xi_{\varepsilon}^{2}(x, 0) d x \\
= & C \int_{\mathbb{R}_{+}^{n+1}} z^{a}\left(\left|\nabla \xi\left(x^{\prime}, z\right)\right|^{2} \eta^{2}\left(\varepsilon x_{n}\right)+\varepsilon^{2}\left|\nabla \eta\left(\varepsilon x_{n}\right)\right|^{2} \xi^{2}\left(x^{\prime}, z\right)+2 \varepsilon \eta\left(\varepsilon x_{n}\right) \xi\left(x^{\prime}, z\right) \nabla \eta\left(\varepsilon x_{n}\right) \cdot \nabla \xi\left(x^{\prime}, z\right)\right) d x d z \\
& \quad+\int_{\mathbb{R}^{n}} F^{\prime \prime}\left(\bar{u}\left(x^{\prime}\right)\right) \xi^{2}\left(x^{\prime}, 0\right) \eta^{2}\left(\varepsilon x_{n}\right) d x .
\end{aligned}
$$

Hence, taking the limit as $\varepsilon \rightarrow 0$,

$$
0 \leqslant C \int_{\mathbb{R}_{+}^{n+1}} z^{a}\left|\nabla \xi\left(x^{\prime}, z\right)\right|^{2} d x d z+\int_{\mathbb{R}^{n}} F^{\prime \prime}\left(\bar{u}\left(x^{\prime}\right)\right) \xi^{2}\left(x^{\prime}, 0\right) d x
$$

and so $\bar{u}$ is stable in $\mathbb{R}^{n-1}$. This proves (4.1) for $\bar{u}$ (the case of $\underline{u}$ is similar).

As a consequence of (4.1) and of the classification results in the plane (see in particular Theorem 2.12 in [CS15], or [SV09]), we conclude that $\underline{u}$ and $\bar{u}$ are 1D and monotone. Then, the local minimality is a consequence of Lemma 3.1. 


\section{LOCAL MINIMIZATION BY RANGE CONSTRAinT}

In this section, we point out that perturbations which do not pointwise exceed the limit profiles necessarily increase the energy. For the classical case, this property has been exploited in Theorem 4.5 of [AAC01], Theorem 10.4 of [DG02] and Lemma 2.2 of [FV11]. In the framework of this paper, the result that we need is the following:

Lemma 5.1. Let $s \in(0,1)$ and $u \in C^{2}\left(\mathbb{R}^{n},[-1,1]\right)$ be a solution of $(-\Delta)^{s} u=f(u)$ in $\mathbb{R}^{n}$, with $\partial_{x_{n}} u>0$ in $\mathbb{R}^{n}$. Let

$$
\underline{u}\left(x^{\prime}\right):=\lim _{x_{n} \rightarrow-\infty} u\left(x^{\prime}, x_{n}\right) \quad \text { and } \quad \bar{u}\left(x^{\prime}\right):=\lim _{x_{n} \rightarrow+\infty} u\left(x^{\prime}, x_{n}\right) .
$$

Let also $R>0$ and $\Phi \in C_{0}^{\infty}\left(\mathcal{B}_{R}\right)$ and suppose that

$$
\left(E_{u}+\Phi\right)(X) \in\left[E_{\underline{u}}(X), E_{\bar{u}}(X)\right] \quad \text { for any } X \in \mathbb{R}_{+}^{n+1} \text {. }
$$

Then $\mathcal{E}_{\mathcal{B}_{R}}\left(E_{u}\right) \leqslant \mathcal{E}_{\mathcal{B}_{R}}\left(E_{u}+\Phi\right)$.

Proof. The argument is by contradiction. We suppose that there exist $R>0$ and a perturbation $\Phi \in$ $C_{0}^{\infty}\left(\mathcal{B}_{R}\right)$ with

and such that

$$
\left(E_{u}+\Phi\right)(X) \in\left[E_{\underline{u}}(X), E_{\bar{u}}(X)\right] \quad \text { for any } X \in \mathbb{R}_{+}^{n+1}
$$

$$
\mathcal{E}_{\mathcal{B}_{R}}\left(E_{u}+\Phi\right)<\mathcal{E}_{\mathcal{B}_{R}}\left(E_{u}\right)
$$

That is, letting $\varphi(x):=\Phi(x, 0)$, by Proposition 2.3 ,

$$
\begin{aligned}
0 & >\frac{1}{2} \int_{\mathbb{R}_{+}^{n+1}} z^{a}\left(\left|\nabla\left(E_{u}+\Phi\right)(x, z)\right|^{2}-\left|\nabla E_{u}(x, z)\right|^{2}\right) d x d z+\int_{B_{R}}(F((u+\varphi)(x))-F(u(x))) d x \\
& =\frac{1}{2} \iint_{\mathbb{R}^{2 n}} \frac{|(u+\varphi)(x)-(u+\varphi)(y)|^{2}-|u(x)-u(y)|^{2}}{|x-y|^{n+2 s}} d x d y+\int_{B_{R}}(F((u+\varphi)(x))-F(u(x))) d x \\
& =\mathcal{F}_{B_{R}}(u+\varphi)-\mathcal{F}_{B_{R}}(u) .
\end{aligned}
$$

Therefore, there exists a perturbation $w_{o}:=u+\varphi_{o}$ of $u$, with $\varphi_{o} \in C_{0}^{\infty}\left(B_{R}\right)$, such that

$$
w_{o}(x) \in\left[\underline{u}\left(x^{\prime}\right), \bar{u}\left(x^{\prime}\right)\right] \quad \text { for any } x \in \mathbb{R}^{n}
$$

and

$$
\mathcal{F}_{B_{R}}\left(w_{o}\right)=\inf _{\substack{\varphi \in C_{0}^{\infty}\left(B_{R}\right) \\(u+\varphi)(x) \in\left[\underline{u}\left(x^{\prime}\right), \bar{u}\left(x^{\prime}\right)\right]}} \mathcal{F}_{B_{R}}(u+\varphi)<\mathcal{F}_{B_{R}}(u)
$$

As a consequence, by taking energy perturbations, we see that $(-\Delta)^{s} w_{o}=f^{\prime}\left(w_{o}\right)$ inside $\{x \in$ $\mathbb{R}^{n}$ s.t. $\left.w_{o}(x) \in\left[\underline{u}\left(x^{\prime}\right), \bar{u}\left(x^{\prime}\right)\right]\right\}$. In addition, if $w\left(x_{o}\right)=\bar{u}\left(x_{o}\right)$, then $(-\Delta)^{s} w_{o}\left(x_{o}\right) \leqslant f^{\prime}\left(w_{o}\left(x_{o}\right)\right)$. Similarly, if $w\left(x_{o}\right)=\underline{u}\left(x_{o}\right)$, then $(-\Delta)^{s} w_{o}\left(x_{o}\right) \geqslant f^{\prime}\left(w_{o}\left(x_{o}\right)\right)$.

Now we claim that strict inequalities hold in (5.1), namely

$$
w_{o}(x) \in\left(\underline{u}\left(x^{\prime}\right), \bar{u}\left(x^{\prime}\right)\right) \quad \text { for any } x \in \mathbb{R}^{n} .
$$

To check this, suppose by contradiction, for instance, that there exists $x_{o} \in \mathbb{R}^{n}$ such that $w_{o}\left(x_{o}\right)=$ $\bar{u}\left(x_{o}^{\prime}\right)$. Then, the function $\zeta(x):=\bar{u}\left(x^{\prime}\right)-w_{o}(x)$ has a minimum at $x_{o}$. Accordingly,

$$
0 \geqslant(-\Delta)^{s} \zeta\left(x_{o}\right) \geqslant f\left(\bar{u}\left(x_{o}^{\prime}\right)\right)-f^{\prime}\left(w_{o}\left(x_{o}\right)\right)=0 .
$$

This gives that $\zeta$ must vanish identically, and thus that $w_{o}$ is identically equal to $\bar{u}$. Then, taking $\bar{x} \in$ $\mathbb{R}^{n} \backslash B_{R}$, we have that

$$
\bar{u}\left(\bar{x}^{\prime}\right)=w_{o}(\bar{x})=u(\bar{x})<\bar{u}\left(\bar{x}^{\prime}\right) .
$$

This is a contradiction, and therefore (5.3) is established.

From (5.3), it follows that

$$
\max _{\overline{B_{R}}}\left(w_{o}-\bar{u}\right)<0
$$


Now, we let $w_{k}(x):=u\left(x+k e_{n}\right)$. We claim that there exists $\bar{k} \in \mathbb{N}$ such that

$$
w_{\bar{k}}(x)>w_{o}(x) \text { for any } x \in \mathbb{R}^{n} \text {. }
$$

To prove this, we argue by contradiction and suppose that for any $k \in \mathbb{N}$ there exists $x_{k} \in \mathbb{R}^{n}$ such that $w_{k}\left(x_{k}\right) \leqslant w_{o}\left(x_{k}\right)$. Then, $x_{k} \in B_{R}$ (otherwise $\left.w_{o}\left(x_{k}\right)=u\left(x_{k}\right)<u\left(x_{k}+k e_{n}\right)=w_{k}\left(x_{k}\right)\right)$. Accordingly, up to a subsequence, we may suppose that $x_{k} \rightarrow \bar{x}$, for some $\bar{x} \in \overline{B_{R}}$ as $k \rightarrow+\infty$. Consequently,

$0 \leqslant \lim _{k \rightarrow+\infty} w_{o}\left(x_{k}\right)-w_{k}\left(x_{k}\right)=w_{o}(\bar{x})-\lim _{k \rightarrow+\infty} u\left(x_{k}-k e_{n}\right)=w_{o}(\bar{x})-\lim _{k \rightarrow+\infty} u\left(\bar{x}-k e_{n}\right)=w_{o}(\bar{x})-\bar{u}\left(\bar{x}^{\prime}\right)$.

But this inequality is in contradiction with (5.3) and thus we have proved (5.4).

Now, starting from (5.4), we can reduce $k$ till a touching between $w_{k}$ and $w_{o}$ occurs. That is, we define $k_{\star}:=\inf \left\{k \in[0, \bar{k}]\right.$ s.t. $\left.w_{k}>w_{o}\right\}$. We claim that

$$
k_{\star}=0 .
$$

Once again, suppose not. Then, the function $v:=w^{k_{\star}}-w_{o}$ would satisfy $v \geqslant 0$ in $\mathbb{R}^{n}$, with $v(\tilde{x})=0$, for some $\tilde{x} \in \overline{B_{R}}$. As a consequence,

$$
0 \geqslant(-\Delta)^{s} v(\tilde{x})=f\left(w^{k_{\star}}(\tilde{x})\right)-f(w(\tilde{x}))=0,
$$

which implies that $v$ vanishes identically. In particular, fixing $x_{\star}$ outside $B_{R}$, we would have that

$$
0=v\left(x_{\star}\right)=w^{k_{\star}}\left(x_{\star}\right)-w_{o}\left(x_{\star}\right)=u\left(x_{\star}+k_{\star} e_{n}\right)-u\left(x_{\star}\right)>0 .
$$

This contradiction completes the proof of (5.5).

Now, in view of (5.5), we obtain that, for any $x \in \mathbb{R}^{n}$,

$$
w_{o}(x) \leqslant \lim _{k \searrow 0} w_{k}(x)=\lim _{k \searrow 0} u\left(x+k e_{n}\right)=u(x) .
$$

Similarly, one can prove that $w_{o}(x) \geqslant u(x)$. Therefore, $w_{o}$ and $u$ must coincide and so $\mathcal{F}_{B_{R}}\left(w_{o}\right)=$ $\mathcal{F}_{B_{R}}(u)$. But this fact is in contradiction with (5.2) and so we have completed the proof of Lemma 5.1 ,

\section{LOCAL MINIMIZATION PROPERTIES INHERITED FROM THOSE OF THE PROFILES AT INFINITY}

In this section, we show that if the profiles at infinity are local minimizers, then so is the original solution. In the classical case of the Laplacian, this property was discussed, for instance, in Proposition 2.3 of [FV11. In our setting, the result that we need is the following (and it uses the pivotal definition of extended local minimizer in Definition [2.2):

Lemma 6.1. Let $s \in(0,1)$ and $u \in C^{2}\left(\mathbb{R}^{n},[-1,1]\right)$ be a solution of $(-\Delta)^{s} u=f(u)$ in $\mathbb{R}^{n}$, with $\partial_{x_{n}} u>0$ in $\mathbb{R}^{n}$. Let

$$
\underline{u}\left(x^{\prime}\right):=\lim _{x_{n} \rightarrow-\infty} u\left(x^{\prime}, x_{n}\right) \quad \text { and } \quad \bar{u}\left(x^{\prime}\right):=\lim _{x_{n} \rightarrow+\infty} u\left(x^{\prime}, x_{n}\right)
$$

and suppose that $\underline{u}$ and $\bar{u}$ are local minimizers (in $\mathbb{R}^{n-1}$ ). Then, $E_{u}$ is an extended local minimizer (in $\mathbb{R}_{+}^{n+1}$ ) and $u$ is a local minimizer $\left(\right.$ in $\left.\mathbb{R}^{n}\right)$.

Proof. Our goal is to show that $E_{u}$ is an extended local minimizer in the sense of Definition 2.2 (from this, we also obtain that $u$ is a local minimizer, thanks to Proposition 2.5). To this aim, fixed $x_{n} \in \mathbb{R}$, we use the following "slicing notation" for a domain $\Omega \subset \mathbb{R}^{n+1}$ : we let

$$
\Omega^{x_{n}}:=\left\{\left(x^{\prime}, z\right) \in \mathbb{R}^{n-1} \times \mathbb{R} \text { s.t. }\left(x^{\prime}, x_{n}, z\right) \in \Omega\right\} .
$$


Also, the function $\bar{u}: \mathbb{R}^{n-1} \rightarrow \mathbb{R}$ can be seen as a function on $\mathbb{R}^{n}$, by defining $\bar{u}_{\star}\left(x^{\prime}, x_{n}\right):=\bar{u}\left(x^{\prime}\right)$ and so we can consider its $a$-harmonic extension $E_{\bar{u}_{\star}}$. Given $R>0$ and $\Phi \in C_{0}^{\infty}\left(\mathcal{B}_{R}\right)$, with $\varphi(x):=\Phi(x, 0)$, we also define $\Phi_{x_{n}}\left(x^{\prime}, z\right):=\Phi\left(x^{\prime}, x_{n}, z\right)$ and $\varphi_{x_{n}}\left(x^{\prime}\right):=\varphi\left(x^{\prime}, x_{n}\right)$. Hence we have that

$$
\begin{aligned}
\mathcal{E}_{\mathcal{B}_{R}}\left(E_{\bar{u}_{\star}}+\Phi\right)= & \frac{1}{2} \int_{\mathcal{B}_{R}^{+}} z^{a}\left|\nabla\left(E_{\bar{u}_{\star}}+\Phi\right)(x, z)\right|^{2} d x d z+\int_{B_{R}} F\left(\bar{u}\left(x^{\prime}\right)+\varphi(x)\right) d x \\
\geqslant & \int_{\mathbb{R}}\left[\frac{1}{2} \int_{\left(B_{R}\right)_{x_{n}} \times(0, R)} z^{a}\left|\nabla_{\left(x^{\prime}, z\right)} E_{\bar{u}}\left(x^{\prime}, z\right)+\nabla_{\left(x^{\prime}, z\right)} \Phi_{x_{n}}\left(x^{\prime}, z\right)\right|^{2} d x^{\prime} d z\right. \\
& \left.+\int_{\left(B_{R}\right)_{x_{n}}} F\left(\bar{u}\left(x^{\prime}\right)+\varphi_{x_{n}}(x)\right) d x^{\prime}\right] d x_{n}
\end{aligned}
$$

Also, since $\bar{u}$ is a local minimizer in $\mathbb{R}^{n-1}$, we have that $E_{\bar{u}}$ is an extended local minimizer in $\mathbb{R}^{n-1} \times$ $(0,+\infty)$, thanks to Proposition 2.5, and therefore

$$
\begin{aligned}
& \frac{1}{2} \int_{\left(B_{R}\right)_{x_{n}} \times(0, R)} z^{a}\left|\nabla_{\left(x^{\prime}, z\right)} E_{\bar{u}}\left(x^{\prime}, z\right)+\nabla_{\left(x^{\prime}, z\right)} \Phi_{x_{n}}\left(x^{\prime}, z\right)\right|^{2} d x^{\prime} d z+\int_{\left(B_{R}\right)_{x_{n}}} F\left(\bar{u}\left(x^{\prime}\right)+\varphi_{x_{n}}(x)\right) d x^{\prime} \\
\geqslant & \frac{1}{2} \int_{\left(B_{R}\right)_{x_{n}} \times(0, R)} z^{a}\left|\nabla_{\left(x^{\prime}, z\right)} E_{\bar{u}}\left(x^{\prime}, z\right)\right|^{2} d x^{\prime} d z+\int_{\left(B_{R}\right)_{x_{n}}} F\left(\bar{u}\left(x^{\prime}\right)\right) d x^{\prime} .
\end{aligned}
$$

By inserting this inequality into (6.1), we obtain that $\mathcal{E}_{\mathcal{B}_{R}}\left(E_{\bar{u}_{\star}}+\Phi\right) \geqslant \mathcal{E}_{\mathcal{B}_{R}}\left(E_{\bar{u}_{\star}}\right)$. That is,

$$
E_{\bar{u}_{\star}} \text { is an extended local minimizer in } \mathbb{R}_{+}^{n+1} \text {. }
$$

Similarly, one can define $\underline{u}_{\star}\left(x^{\prime}, x_{n}\right):=\underline{u}\left(x^{\prime}\right)$ and conclude that

$$
E_{\underline{u}_{\star}} \text { is an extended local minimizer in } \mathbb{R}_{+}^{n+1} \text {. }
$$

Now, given $R>0$ and $\Psi \in C_{0}^{\infty}\left(\mathcal{B}_{R}\right)$, with $\psi(x):=\Psi(x, 0)$, we consider the perturbation $E_{u}+\Psi$. We define

$$
\begin{aligned}
\alpha(X):=\left\{\begin{array}{cl}
E_{\bar{u}_{\star}}(X) & \text { if }\left(E_{u}+\Psi\right)(X) \leqslant E_{\bar{u}_{\star}}(X), \\
\left(E_{u}+\Psi\right)(X) & \text { if }\left(E_{u}+\Psi\right)(X)>E_{\bar{u}_{\star}}(X),
\end{array}\right. \\
\beta(X):=\left\{\begin{array}{cc}
E_{\bar{u}_{\star}}(X) & \text { if }\left(E_{u}+\Psi\right)(X)>E_{\bar{u}_{\star}}(X), \\
E_{\underline{u}_{\star}}(X) & \text { if }\left(E_{u}+\Psi\right)(X)<E_{u_{\star}}(X), \\
\left(E_{u}+\Psi\right)(X) & \text { if }\left(E_{u}+\Psi\right)(X) \in\left[E_{\underline{u}_{\star}}(X), E_{\bar{u}_{\star}}(X)\right],
\end{array}\right. \\
\text { and } \gamma(X):=\left\{\begin{array}{cl}
E_{\underline{u}_{\star}}(X) & \text { if }\left(E_{u}+\Psi\right)(X) \geqslant E_{\underline{u}_{\star}}(X), \\
\left(E_{u}+\Psi\right)(X) & \text { if }\left(E_{u}+\Psi\right)(X)<E_{\underline{u}_{\star}}(X) .
\end{array}\right.
\end{aligned}
$$

By (6.2), we have that

$$
\mathcal{E}_{\left\{E_{u}+\Psi>E_{\bar{u}_{\star}}\right\}}\left(E_{\bar{u}_{\star}}\right) \leqslant \mathcal{E}_{\left\{E_{u}+\Psi>E_{\left.\bar{u}_{\star}\right\}}\right.}(\alpha) .
$$

Similarly, by (6.3), we have that

$$
\mathcal{E}_{\left\{E_{u}+\Psi<E_{\underline{u}_{*}}\right\}}\left(E_{\underline{u}_{\star}}\right) \leqslant \mathcal{E}_{\left\{E_{u}+\Psi<E_{\underline{u}_{\star}}\right\}}(\gamma) .
$$

In addition, from Lemma 5.1,

$$
\mathcal{E}_{\mathcal{B}_{R}}\left(E_{u}\right) \leqslant \mathcal{E}_{\mathcal{B}_{R}}(\beta)
$$


Notice also that $\left\{E_{u}+\Psi>E_{\bar{u}_{\star}}\right\}$ and $\left\{E_{u}+\Psi<E_{\underline{u}_{\star}}\right\}$ are subset of $\mathcal{B}_{R}$. Thus, using (6.4), (6.5) and (6.6),

$$
\begin{aligned}
& \mathcal{E}_{\mathcal{B}_{R}}\left(E_{u}+\Psi\right)=\mathcal{E}_{\left\{E_{u}+\Psi>E_{\bar{u}_{\star}}\right\}}\left(E_{u}+\Psi\right)+\mathcal{E}_{\left\{E_{u}+\Psi<E_{\underline{u}_{*}}\right\}}\left(E_{u}+\Psi\right)+\mathcal{E}_{\mathcal{B}_{R} \cap\left\{E_{\underline{u}_{*}} \leqslant E_{u}+\Psi \leqslant E_{\bar{u}_{\star}}\right\}}\left(E_{u}+\Psi\right) \\
& =\mathcal{E}_{\left\{E_{u}+\Psi>E_{\bar{u}_{\star}}\right\}}(\alpha)+\mathcal{E}_{\left\{E_{u}+\Psi<E_{\underline{u}_{*}}\right\}}(\gamma)+\mathcal{E}_{\mathcal{B}_{R} \cap\left\{E_{\underline{u}_{*}} \leqslant E_{u}+\Psi \leqslant E_{\bar{u}_{\star}}\right\}}(\beta) \\
& \geqslant \mathcal{E}_{\left\{E_{u}+\Psi>E_{\bar{u}_{\star}}\right\}}\left(E_{\bar{u}_{\star}}\right)+\mathcal{E}_{\left\{E_{u}+\Psi<E_{\underline{u}_{*}}\right\}}\left(E_{\underline{u}_{\star}}\right)+\mathcal{E}_{\mathcal{B}_{R} \cap\left\{E_{\underline{u}_{\star}} \leqslant E_{u}+\Psi \leqslant E_{\left.\bar{u}_{\star}\right\}}\right.}(\beta) \\
& =\mathcal{E}_{\left\{E_{u}+\Psi>E_{\bar{u}_{\star}}\right\}}(\beta)+\mathcal{E}_{\left\{E_{u}+\Psi<E_{\underline{u}_{*}}\right\}}(\beta)+\mathcal{E}_{\mathcal{B}_{R} \cap\left\{E_{\underline{u}_{*}} \leqslant E_{u}+\Psi \leqslant E_{\bar{u}_{\star}}\right\}}(\beta) \\
& =\mathcal{E}_{\mathcal{B}_{R}}(\beta) \\
& \geqslant \mathcal{E}_{\mathcal{B}_{R}}\left(E_{u}\right)
\end{aligned}
$$

This shows that $E_{u}$ is an extended local minimizer, as desired.

\section{Proof of Theorem 1.1}

By Lemma 4.1, we know that both $\underline{u}$ and $\bar{u}$ are local minimizers. This and Lemma 6.1 imply that $u$ is a local minimizer. Therefore, by Lemma 8.1 in [DSV16], we have that $u_{\varepsilon}(x):=u(x / \varepsilon)$ approaches, as $\varepsilon \rightarrow 0$, up to subsequences, a step function of the form $\chi_{E}-\chi_{E^{c}}$, and the level sets of $u_{\varepsilon}$ approach $\partial E$ locally uniformly. We claim that

$$
E \text { is a halfspace. }
$$

In a sense, this claim is a version of the Bernstein-type result in [FV17], for which we provide a complete and independent proof, by arguing as follows. To prove (7.1), it is enough to show that

either $E$ is contained in a halfspace, or it contains a halfspace,

see e.g. Lemma 8.3 in [DSV16], hence we focus on the proof of (7.2). To this end, we distinguish two cases,

$$
\text { either } \underline{u}=-1 \text { and } \bar{u}=1 \text {, }
$$

or

at least one between $\underline{u}$ and $\bar{u}$ is non-constant.

In case (7.3), we can exploit Theorem 1.4 in [DSV16] and obtain in particular that (7.2) holds true, so we focus on case (7.4) and we suppose that $\underline{u}$ is non-constant (the case in which $\bar{u}$ is non-constant is similar).

Then, setting $\underline{u}_{\varepsilon}(x):=\underline{u}(x / \varepsilon)$ we have that $\underline{u}_{\varepsilon}$ approaches, as $\varepsilon \rightarrow 0$, up to subsequences, a step function of the form $\chi_{\underline{E}}-\chi_{\underline{E}^{c}}$. Since $\underline{u}$ is a non-constant $1 \mathrm{D}$ function, we have that $\underline{E}$ is a halfspace. In addition, a.e. $x \in \mathbb{R}^{n}$,

$$
\left(\chi_{\underline{E}}-\chi_{\underline{E}^{c}}\right)(x)=\lim _{\varepsilon \searrow 0} \underline{u}(x / \varepsilon) \leqslant \lim _{\varepsilon \searrow 0} u(x / \varepsilon)=\left(\chi_{E}-\chi_{E^{c}}\right)(x),
$$

and consequently $\underline{E} \subseteq E$. This proves (7.2), and so (7.1).

Hence, $u$ is necessarily 1D, thanks to (7.1) and Theorem 1.2 in [DSV16].

\section{The CASE OF TWO-DIMENSIONAL PROFILES AT INFINITY}

For completeness, in relation with the work in CV13], we observe that our arguments provide also the following variation of Theorem 1.1;

Theorem 8.1. Let $s \in\left(0, \frac{1}{2}\right)$ and $u \in C^{2}\left(\mathbb{R}^{n},[-1,1]\right)$ be a solution of $(-\Delta)^{s} u=u-u^{3}$ in $\mathbb{R}^{n}$, with $\partial_{x_{n}} u>0$ in $\mathbb{R}^{n}$.

Let

$$
\underline{u}\left(x^{\prime}\right):=\lim _{x_{n} \rightarrow-\infty} u\left(x^{\prime}, x_{n}\right) \quad \text { and } \quad \bar{u}\left(x^{\prime}\right):=\lim _{x_{n} \rightarrow+\infty} u\left(x^{\prime}, x_{n}\right) .
$$


Assume that (possibly after a rotation) $\underline{u}$ and $\bar{u}$ depend on at most two Euclidean variables (not necessarily the same).

Then $u$ is a local minimizer.

Moreover, if $n \leqslant 8$, there exists $s(n) \in\left[0, \frac{1}{2}\right)$ such that if $s \in\left(s(n), \frac{1}{2}\right)$ then $u$ is $1 D$.

The proof of Theorem 8.1] shares the point of view taken in [FSV08] and [FV11], and follows the same lines as that of Theorem 1.1, with the modifications listed here below:

- By following verbatim the proof of Lemma 4.1, and exploiting that $\underline{u}$ and $\bar{u}$ are stable twodimensional solutions, one obtains that they are local minimizers and 1D;

- From this and Lemma 6.1, one deduces the first claim in Theorem 8.1,

- The second claim in Theorem 8.1 follows from the first claim and the argument in Section 7 (in this framework, for $s$ large enough, one can exploit Theorem 1.6 of [DSV16] in place of Theorem 1.4 of [DSV16]).

\section{REFERENCES}

[AAC01] Giovanni Alberti, Luigi Ambrosio, and Xavier Cabré. On a long-standing conjecture of E. De Giorgi: symmetry in 3D for general nonlinearities and a local minimality property. Acta Appl. Math., 65(1-3):933, 2001. ISSN 0167-8019. URL \protect \vrulewidth0pt\protect \href $\{\mathrm{http}: / / \mathrm{dx}$.doi.org/10.1023/ A : 1010602715526\} \{http://dx.doi.org/10.1023/A: 1010602715526\}. Special issue dedicated to Antonio Avantaggiati on the occasion of his 70th birthday.

[BPSV14] Begoña Barrios, Ireneo Peral, Fernando Soria, and Enrico Valdinoci. A Widder's type theorem for the heat equation with nonlocal diffusion. Arch. Ration. Mech. Anal., 213(2):629-650, 2014. ISSN 0003-9527. URL \protect\vrulewidth0pt\protect \href\{http://dx.doi.org/10.1007/ s00205-014-0733-1\}\{http://dx.doi.org/10.1007/s00205-014-0733-1\}.

[Buc16] Claudia Bucur. Some observations on the Green function for the ball in the fractional Laplace framework. Commun. Pure Appl. Anal., 15(2):657-699, 2016. ISSN 1534-0392. URL $\backslash$ protect \vrulewidthopt \protect \href \{http://dx.doi.org/10.3934/cpaa.2016.15.657\}\{http: //dx.doi.org/10.3934/cpaa.2016.15.657\}.

[BV16] Claudia Bucur and Enrico Valdinoci. Nonlocal diffusion and applications, volume 20 of Lecture Notes of the Unione Matematica Italiana. Springer, [Cham]; Unione Matematica Italiana, Bologna, 2016. ISBN 978-3-319-28738-6; 978-3-319-28739-3. xii+155 pp. URL \protect \vrulewidth0pt \protect \href $\{\mathrm{http}$ : //dx.doi.org/10.1007/978-3-319-28739-3\}\{http://dx.doi.org/10.1007/978-3-319-28739-3\}.

[CC10] Xavier Cabré and Eleonora Cinti. Energy estimates and 1-D symmetry for nonlinear equations involving the half-Laplacian. Discrete Contin. Dyn. Syst., 28(3):1179-1206, 2010. ISSN 1078-0947. URL \protect \vrulewidth0pt \protect \href \{http://dx.doi.org/10.3934/dcds.2010.28. 1179\}\{http://dx.doi.org/10.3934/dcds.2010.28.1179\}.

[CC14] Xavier Cabré and Eleonora Cinti. Sharp energy estimates for nonlinear fractional diffusion equations. Calc. Var. Partial Differential Equations, 49(1-2):233-269, 2014. ISSN 0944-2669. URL $\backslash$ protect \vrulewidth0pt \protect \href \{http://dx.doi.org/10.1007/s00526-012-0580-6\}\{http: //dx.doi.org/10.1007/s00526-012-0580-6\}.

[CCS17] Xavier Cabré, Eleonora Cinti, and Joaquim Serra. Stable nonlocal phase transition. Preprint, 2017.

[CRS10] L. Caffarelli, J.-M. Roquejoffre, and O. Savin. Nonlocal minimal surfaces. Comm. Pure Appl. Math., 63(9): 1111-1144, 2010. ISSN 0010-3640. URL \protect \vrulewidth0pt\protect \href\{http://dx.doi.org/ 10.1002/cpa.20331\} \{http://dx.doi.org/10.1002/cpa.20331\}.

[CS07] Luis Caffarelli and Luis Silvestre. An extension problem related to the fractional Laplacian. Comm. Partial Differential Equations, 32(7-9):1245-1260, 2007. ISSN 0360-5302. URL https://doi-org.pros.lib. unimi.it:2050/10.1080/03605300600987306.

[CS14] Xavier Cabré and Yannick Sire. Nonlinear equations for fractional Laplacians, I: Regularity, maximum principles, and Hamiltonian estimates. Ann. Inst. H. Poincaré Anal. Non Linéaire, 31(1):23-53, 2014. ISSN 0294-1449. URL \protect\vrulewidth0pt\protect \href\{http://dx.doi.org/10.1016/j. anihpc.2013.02.001\}\{http://dx.doi.org/10.1016/j.anihpc.2013.02.001\}.

[CS15] Xavier Cabré and Yannick Sire. Nonlinear equations for fractional Laplacians II: Existence, uniqueness, and qualitative properties of solutions. Trans. Amer. Math. Soc., 367(2):911-941, 2015. ISSN 0002-9947. URL \protect \vrulewidth0pt\protect \href\{http://dx.doi.org/10.1090/ S0002-9947-2014-05906-0\}\{http://dx.doi.org/10.1090/S0002-9947-2014-05906-0\}. 
[CSM05] Xavier Cabré and Joan Solà-Morales. Layer solutions in a half-space for boundary reactions. Comm. Pure Appl. Math., 58(12):1678-1732, 2005. ISSN 0010-3640. URL \protect \vrulewidth0pt\protect\ href \{http://dx.doi.org/10.1002/cpa.20093\}\{http://dx.doi.org/10.1002/cpa.20093\}.

[CV13] Luis Caffarelli and Enrico Valdinoci. Regularity properties of nonlocal minimal surfaces via limiting arguments. Adv. Math., 248:843-871, 2013. ISSN 0001-8708. URL \protect\vrulewidth0pt\protect href\{http://dx.doi.org/10.1016/j.aim.2013.08.007\}\{http://dx.doi.org/10.1016/j.aim. 2013. 08.007\}.

[DFV17] Serena Dipierro, Alberto Farina, and Enrico Valdinoci. A three-dimensional symmetry result for a phase transition equation in the genuinely nonlocal regime. ArXiv e-prints, April 2017.

[DG79] Ennio De Giorgi. Convergence problems for functionals and operators. In Proceedings of the International Meeting on Recent Methods in Nonlinear Analysis (Rome, 1978), pages 131-188. Pitagora, Bologna, 1979.

[DG02] Donatella Danielli and Nicola Garofalo. Properties of entire solutions of non-uniformly elliptic equations arising in geometry and in phase transitions. Calc. Var. Partial Differential Equations, 15(4): 451-491, 2002. ISSN 0944-2669. URL \protect \vrulewidth0pt\protect \href \{http://dx.doi.org/10. 1007/s005260100133\}\{http://dx.doi.org/10.1007/s005260100133\}.

[DSV16] Serena Dipierro, Joaquim Serra, and Enrico Valdinoci. Improvement of flatness for nonlocal phase transitions. ArXiv e-prints, November 2016.

[FSV08] Alberto Farina, Berardino Sciunzi, and Enrico Valdinoci. Bernstein and De Giorgi type problems: new results via a geometric approach. Ann. Sc. Norm. Super. Pisa Cl. Sci. (5), 7(4):741-791, 2008. ISSN 0391$173 \mathrm{X}$.

[FV11] Alberto Farina and Enrico Valdinoci. 1D symmetry for solutions of semilinear and quasilinear elliptic equations. Trans. Amer. Math. Soc., 363(2):579-609, 2011. ISSN 0002-9947. URL \protect \ vrulewidth0pt \protect \href \{http://dx.doi.org/10.1090/S0002-9947-2010-05021-4\}\{http: //dx.doi.org/10.1090/S0002-9947-2010-05021-4\}.

[FV17] Alessio Figalli and Enrico Valdinoci. Regularity and Bernstein-type results for nonlocal minimal surfaces. J. Reine Angew. Math., 729:263-273, 2017. ISSN 0075-4102. URL https://doi-org.pros.lib.unimi.it: 2050/10.1515/crelle-2015-0006.

[Sav16] Ovidiu Savin. Rigidity of minimizers in nonlocal phase transitions. ArXiv e-prints, October 2016.

[SV09] Yannick Sire and Enrico Valdinoci. Fractional Laplacian phase transitions and boundary reactions: a geometric inequality and a symmetry result. J. Funct. Anal., 256(6):1842-1864, 2009. ISSN 0022-1236. URL \protect \vrulewidth0pt\protect \href\{http://dx.doi.org/10.1016/j.jfa.2009. $01.020\}\{$ http://dx.doi.org/10.1016/j.jfa.2009.01.020\}.

[SV12] Ovidiu Savin and Enrico Valdinoci. Г-convergence for nonlocal phase transitions. Ann. Inst. H. Poincaré Anal. Non Linéaire, 29(4):479-500, 2012. ISSN 0294-1449. URL \protect\vrulewidth0pt \protect\ href \{http://dx.doi.org/10.1016/j.anihpc.2012.01.006\}\{http://dx.doi.org/10.1016/j.anihpc. $2012.01 .006\}$.

[SV13] Ovidiu Savin and Enrico Valdinoci. Some monotonicity results for minimizers in the calculus of variations. J. Funct. Anal., 264(10):2469-2496, 2013. ISSN 0022-1236. URL \protect\vrulewidth0pt \protect \ href\{http://dx.doi.org/10.1016/j.jfa.2013.02.005\}\{http://dx.doi.org/10.1016/j.jfa.2013. $02.005\}$.

[SV14] Ovidiu Savin and Enrico Valdinoci. Density estimates for a variational model driven by the Gagliardo norm. J. Math. Pures Appl. (9), 101(1):1-26, 2014. ISSN 0021-7824. URL \protect\ vrulewidth0pt \protect \href \{http://dx.doi.org/10.1016/j.matpur.2013.05.001\}\{http:

//dx.doi.org/10.1016/j.matpur.2013.05.001\}.

[VSS06] Enrico Valdinoci, Berardino Sciunzi, and Vasile Ovidiu Savin. Flat level set regularity of $p$ Laplace phase transitions. Mem. Amer. Math. Soc., 182(858):vi+144, 2006. ISSN 0065-9266. URL \protect\vrulewidth0pt\protect \href\{http://dx.doi.org/10.1090/memo/0858\}\{http://dx. doi.org/10.1090/memo/0858\}. 
(Serena Dipierro) Dipartimento di Matematica, Università Degli studi di Milano, Via Saldini 50, 20133 Milan, Italy

E-mail address: serena.dipierro@unimi.it

(Alberto Farina) LAMFA - CNRS UMR 6140 and Faculté des Sciences, Université de Picardie Jules Verne, 33 Rue Saint-Leu, 80039 Amiens CEDEX 1, France

E-mail address: alberto.farina@u-picardie.fr

(Enrico Valdinoci) School of Mathematics and Statistics, University of Melbourne, 813 Swanston Street, Parkville ViC 3010, Australia, and Istituto di Matematica Applicata e Tecnologie Informatiche, Consiglio Nazionale delle Ricerche, Via Ferrata 1, 27100 Pavia, italy, and Dipartimento di Matematica, Università degli studi di Milano, Via Saldini 50, 20133 Milan, Italy

E-mail address: enrico@mat.uniroma3.it 